\title{
Transmission Problems for Parabolic Operators on Polygonal Domains and Applications to the Finite Element Method
}

\author{
Yajie Zhang $^{1}$ - Anna L. Mazzucato ${ }^{2}$ (D)
}

Received: 5 March 2021 / Revised: 27 October 2021 / Accepted: 2 November 2021 /

Published online: 17 December 2021

(c) The Author(s), under exclusive licence to Springer Science+Business Media LLC, part of Springer Nature 2021

\begin{abstract}
We study linear parabolic equations $\partial_{t} u+L u=f$, where $L=-\operatorname{div}(A \nabla)$ is a second-order strongly elliptic operator, on non-smooth two-dimensional bounded domains. The domain is polygonal and not assumed to be convex. The coefficient matrix $A$ is piecewise smooth and exhibits jump discontinuities across a finite number of piecewise smooth curves, collectively denoted the interface. We assume mixed Dirichlet-Neumann boundary conditions and standard transmission conditions at the interface. Under some additional assumptions, we establish well-posedness of the initial-value problem using suitable weighted Sobolev spaces. The solution admits a decomposition $u=u_{\text {reg }}+w_{\mathrm{s}}$, into a function $u_{\text {reg }}$ that belongs to the weighted Sobolev space and a function $w_{\mathrm{s}}$ that is locally constant near the vertices, thus proving wellposedness in an augmented space. We use the theoretical analysis to devise graded meshes that give quasi-optimal rates of convergence for a fully discrete scheme that utilizes finite elements on a space grid and finite differences in time.
\end{abstract}

Keywords Parabolic operators · Transmission · Mixed boundary-value problems · Polygonal domains · Finite element method

Mathematics Subject Classification $35 \mathrm{~K} 10 \cdot 35 \mathrm{~K} 45 \cdot 65 \mathrm{M} 60$

$凶$ Anna L. Mazzucato

alm24@psu.edu

Yajie Zhang

z0005149@zuel.edu.cn

1 School of Mathematics and Statistics, Zhongnan University of Economy and Law, 182\# Nanhu Avenue, East Lake High-tech Development Zone, Wuhan 430073, People's Republic of China

2 Department of Mathematics, Penn State University, University Park, PA 16802, USA 


\section{Introduction}

We consider second-order, linear, strongly parabolic operators on non-smooth domains in two space dimensions. We restrict our attention to non-convex polygonal domains. The coefficients are assumed piecewise smooth and globally bounded, but may jump across a finite number of curves, which we collectively denote as the interface. We assume that standard transmission conditions hold at the interface and impose mixed Dirichlet-Neumann boundary conditions. We are interested in the well-posedness and regularity of the initial-boundary-value-transmission problem (IBVTP for short). Parabolic transmission problems arise in a variety of applications to composite materials, where properties vary from one material to the other, for instance heat conduction problems with or without heat loss across interfaces. They also arise in parabolic freeboundary problems, such as in the Stefan problem and in the Mullins-Sekerka model of solidification in binary alloys [43], in the context of non-linear equations.

Transmission problems for elliptic equations have been widely studied in both smooth and non-smooth settings. It is well known that elliptic regularity does not hold in general for non-smooth domains. The singular behavior has been well studied for conical or corner singularities starting with the seminal work of Kondratiev [30]. Among the extensive literature on the subject, we refer to [6,15-19,37,38]. The singularities can be expressed in terms of singular exponents and singular functions, using a local coordinate representation. In particular, if the domain is convex, weak solutions with data in $L^{2}$ still satisfy Sobolev $H^{2}$ regularity. Points where the boundary conditions change behave similarly to geometric singularities at the boundary. In the case where interfaces are present, regularity holds on each side of the interface, when the interface is sufficiently regular and does not touch the boundary of the domain $[13,14,23,31,35,51,52]$. However, in many applications, the interface is itself singular and crosses the boundary. In the elliptic case the problem has been studied using various techniques, including layer potentials for Lipschitz domains and interfaces (we refer to [24-26,29,42] among several works on the subject), and functional analysis techniques for corner domains [6,8,21,22,39,40,44-46,50].

There is significantly less work on regularity for parabolic problems in non-smooth domains and with mixed boundary conditions (see e.g. [20,28] and references therein), and even fewer works for transmission problems (see e.g. [41] and references therein). If the contact angle is a right angle, then reflection techniques can be used $[1,48,49]$. Recently, maximal $L^{\mathrm{p}}$-regularity was established for parabolic transmission problems on smooth domains with a general contact angle [5]. In our work, we investigate parabolic transmission problems in two-dimensional polygonal domains with general polygonal interfaces, using the framework of weighted Sobolev spaces. For elliptic equations, this framework lends itself to studying quasi-optimal convergence rates for the Finite Element Method (FEM for short) using graded meshes (see e.g. [2,9-12,3234]). Even when interfaces are absent, grading is necessary to achieve the optimal rate of convergence (up to constants) near vertices with aperture angle larger than $\pi$, socalled re-entrant corners.

For conforming schemes, such as the standard FEM, one typically exploits a variational formulation of the problem. For elliptic equations, this formulation leads to the invertibility of the forward operator, and hence solvability, via a coercivity property, in 
a natural weighted space. Solvability and higher regularity for other weights can then be inferred by using Fredholm theory and the fact the index of the operator does not change under small perturbations of the weight. This approach has the advantage of not requiring a careful study of the singular functions and exponents, which can be difficult to carry out for variable coefficients operators, especially in higher dimensions. In order to calculate the index of the forward operator, one must account for certain special situations, in particular the presence of the vertices with Neumann boundary conditions on both sides, and singular points along interfaces. In fact, at these vertices the local operator pencil that describes the spectral properties of the forward operator has a larger kernel. These exceptional points can be treated one by one, by adding more singular functions to the regular part of the solution. By regular part, we mean here the part that belongs to the weighted Sobolev space where the solution lies in the case of homogeneous Dirichlet boundary conditions. In our case, because of the range of weights we consider the regular part vanishes at the vertices. In the parabolic case, a main difficulty arises in constructing a basis of functions on which to expand an approximate solution that lies in the same space as the true solution. For instance, one cannot use eigenfunctions of the Laplace operator as a Galerkin basis, as commonly done on smooth domains, since these eigenfunctions do not satisfy the transmission conditions at the interface.

In this work, we also utilize suitable weighted spaces to establish existence and uniqueness of weak solutions to the IBVTP for the operator $L$, and then to establish higher regularity on each side of the interface, provided the data have higher regularity and satisfy certain compatibility conditions to control time derivatives. We employ the regularity results to devise a grading scheme of an initially uniform triangular mesh of the domain $\Omega$. The grading allows to prove quasi-optimal rates of convergence for the FEM, using polynomial elements. In fact, we show that we can use the grading scheme already introduced in [32] for elliptic problems. Our results could, in principle, be extended to 3D polyhedral domains, following in particular the approach in $[8,9]$ for elliptic problems. However, there are additional difficulties in the parabolic case, stemming from needed compatibility conditions between the data and at the interface.

We follow standard notation for function spaces, in particular $H^{s}(\Omega), s>0$, denote the $L^{2}$-based Sobolev space of order $s$, while $H^{-s}(\Omega)$ denotes the dual space to $H_{0}^{s}(\Omega)$, which is the closure in $H^{s}(\Omega)$ of the space of smooth compactly supported functions in $\Omega$. With $\langle$,$\rangle we denote the standard L^{2}$ inner product. Throughout, at times we will use subscript to indicate derivatives, e.g. $u_{t}=\partial_{t} u$.

We conclude the introduction with an outline of the paper. In Sect. 2 we introduce the problem and the geometric set-up. Next, in Sect. 3 we discuss weighted Sobolev spaces and recall the well-posedness theory for elliptic problems. In Sect. 4 we state and prove our main results, which give well-posedness for the parabolic mixed-boundary-valuetransmission problem in weighted spaces, under suitable conditions. Then in Sect. 5 we derive a formula to compute the singular behavior of solutions at the vertices. Lastly, in Sect. 6 we discuss a mixed fully-discrete scheme to numerically solve the parabolic problem and prove quasi-optimal rates of convergence for it, while in Sect. 7 we present some numerical tests to validate the theoretical analysis. 


\section{Preliminaries}

We denote the domain by $\Omega \subset \mathbb{R}^{2}$ and assume that it is partitioned into a finite number of polygonal subdomains $\Omega_{k}, k=1, \ldots, K$ :

$$
\bar{\Omega}=\bigcup_{k=1}^{K} \overline{\Omega_{k}}
$$

We denote $\Gamma:=\cup_{k=1}^{K} \partial \Omega_{k} \backslash \partial \Omega$ and call it the interface. The interface is the union of finitely many piecewise-linear curves and may intersect the boundary of $\Omega$. We denote by $v$ the unit outer normal vector to $\partial \Omega$, which is well defined except at vertices. We also give an orientation on each open sides of $\Gamma$ that is compatible with a choice of orientation on each $\Omega_{k}$, and denote by $v$ the unit normal induced by this orientation, again well defined except at vertices. We assume that the interior aperture angle $\alpha_{Q}$ at each vertex $Q$ satisfies $0<\alpha_{Q}<2 \pi$. Hence, re-entrant corners, where $\alpha_{Q}>\pi$, are allowed. We assume that $\Omega$ is a Lipschitz domain for simplicity, which excludes pathologies such as internal sides and vertices touching a side.

We consider a linear, strongly elliptic operator $L$ in divergence form:

$$
L w:=-\operatorname{div}(A \nabla w)=-\sum_{i, j=1}^{2} \partial_{j}\left(a_{i j} \partial_{i} w\right)
$$

for $w$ a sufficiently regular, real-valued function on $\bar{\Omega}$. The coefficient matrix $A$ is assumed positive definite and bounded on $\bar{\Omega}$, smooth on each subdomain $\overline{\Omega_{k}}$, $k=1, \ldots, K$, and it may have jump discontinuities across $\Gamma$. For simplicity, we assume also that $A$ is symmetric, so $L$ is formally self-adjoint. We restrict ourselves to divergence-form operators to which Theorem 1 directly applies. However, we expect that adding lower-order terms to $L$ may be treated via a perturbation argument.

We decompose the boundary $\partial \Omega$ into two disjoint subsets, $\partial_{\mathrm{D}} \Omega$ and $\partial_{\mathrm{N}} \Omega$, with $\partial_{\mathrm{D}} \Omega$ closed in $\partial \Omega$ with non-empty interior, so $\partial_{\mathrm{N}} \Omega$ is open and possibly empty. We impose Dirichlet boundary conditions on $\partial_{\mathrm{D}} \Omega$ and Neumann boundary conditions on $\partial_{N} \Omega$. We allow for a forcing term $f$, so we reduce to the case of homogeneous boundary conditions. We impose standard transmission conditions at the interface $\Gamma$. We therefore study the following problem for the parabolic operator $\partial_{t}+L$ on a space-time cylinder $\Omega \times(0, T), 0<T \leq \infty$ :

$$
\begin{cases}L u+u_{t}=f, & \text { in } \cup_{k=1}^{K} \Omega_{k} \times(0, T), \\ u=0, & \text { on } \partial_{\mathrm{D}} \Omega \times(0, T), \\ \nabla_{v}^{A} u=0, & \text { on } \partial_{\mathrm{N}} \Omega \times(0, T), \\ u=g, & \text { in } \Omega \times\{t=0\}, \\ u^{+}=u^{-}, \quad \nabla_{v}^{A} u^{+}=\nabla_{v}^{A} u^{-} & \text {on } \Gamma \times(0, T),\end{cases}
$$


Fig. 1 The domain $\Omega$ and interface $\Gamma$

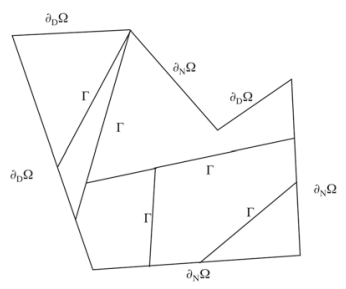

Fig. 2 Two types of vertices

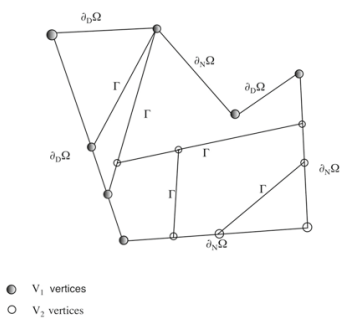

where the unknown $u: \bar{\Omega} \times[0, T) \rightarrow \mathbb{R}, \nabla_{v}^{A}$ denotes the conormal derivative associated with $L$, namely:

$$
\nabla_{v}^{A} u:=v \cdot A \nabla u
$$

and \pm refers to non-tangential limits at the two sides of the interface away from singular points. We define the singular set associated to (3) as follows. $\mathcal{V}$ denotes the set of all geometric vertices of $\Omega_{k}, k=1, \ldots, K$, and of all the points where the boundary conditions change. We note that $\mathcal{V}$ includes points, such as all non-smooth points of $\Gamma$ and the points where $\Gamma$ meets $\partial \Omega$, that are not determined by the geometry of the domain $\Omega$. As in [39], we call such points artificial vertices. The reason for including the artificial vertices is that singularities in the solution at these points is of the same type as at true vertices. At artificial vertices, the interior angle is $\pi$ by definition (see Fig. 1).

For reasons that will be clear later, we subdivide the set of vertices further. Any vertex on $\partial \Omega$ has two sides that lie also on the boundary, although it may also have sides that belong to the interface $\Gamma$. Then, we denote by $\mathcal{V}_{1}$ the set of all boundary vertices such that at least one boundary edge belongs to $\partial_{\mathrm{D}} \Omega$ and we let $\mathcal{V}_{2}$ contain all the remaining vertices. In particular, $\mathcal{V}_{1}$ contains all the points where the boundary conditions change, while $\mathcal{V}_{2}$ contains all interior singular points of the interface $\Gamma$ and all boundary points where both sides belong to $\partial_{N} \Omega$. We refer to the latter type of vertices as Neumann-Neumann vertices (see Fig. 2).

Remark 1 The case of cracks, that is, side internal to $\Omega$, where the aperture angle is $2 \pi$ can also be included, introducing a geometric structure on $\Omega$ that unfolds the boundary. Then, a crack has two distinct sides in this structure. This construction allows also to handle vertices touching a side. We refer to $[8,32]$ for more details on this construction. 
We next discuss the corresponding variational formulation of Problem (3). To this end, we introduce the following bilinear form:

$$
B[u, w]=\int_{\Omega} \sum_{i, j=1}^{2} a_{i j} u_{x_{i}} w_{x_{j}} \mathrm{~d} x,
$$

defined on the Sobolev space $H^{1}(\Omega)$. We also set:

$$
H_{\mathrm{D}}^{1}(\Omega):=\left\{f \in H^{1}(\Omega) \mid u\lfloor\mathrm{D}=0\},\right.
$$

where the restriction of $u$ at the boundary is in trace sense. $\left(H_{\mathrm{D}}^{1}(\Omega)\right)^{\prime}$ denotes the Banach space dual of $H_{\mathrm{D}}^{1}(\Omega)$. This space can be canonically identified with the direct sum $H^{-1}(\Omega) \otimes H^{-1 / 2}\left(\partial_{N} \Omega\right)$, where $H^{-1 / 2}\left(\partial_{N} \Omega\right)=\left(H_{00}^{1 / 2}\left(\partial_{N} \Omega\right)\right)^{\prime}$ and $H_{00}^{1 / 2}$ is the set of all functions in $H^{1 / 2}\left(\partial_{\mathrm{N}} \Omega\right.$ ) that can be extended by zero to $\partial \Omega$ (we refer the reader to $[3,36]$ for further details).

Definition 1 Let $f \in L^{2}\left(0, T ;\left(H_{\mathrm{D}}^{1}(\Omega)\right)^{\prime}\right)$ and let $g \in L^{2}(\Omega)$. The function $u \in$ $L^{2}\left(0, T ; H_{\mathrm{D}}^{1}(\Omega)\right)$ is a weak solution of Problem $(3)$, if $u_{t} \in L^{2}\left(0, T ;\left(H_{\mathrm{D}}^{1}(\Omega)\right)^{\prime}\right)$ and for all $w \in L^{2}\left(0, T ; H_{\mathrm{D}}^{1}(\Omega)\right)$ :

$$
B[u, w]+\left\langle u_{t}, w\right\rangle=\langle f, w\rangle, \quad \text { a.e. } 0<t<T,
$$

and $u(\cdot, 0)=g$ in trace sense.

The weak formulation contains both the homogeneous Neumann boundary condition on $\partial_{N} \Omega$ as well as the standard transmission conditions on $\Gamma$ as the natural conditions. A regular enough solution of Problem (3) is also a weak solution by an application of Green's identities. Since $u$ is assumed in $H^{1}\left(0, T ;\left(H_{\mathrm{D}}^{1}(\Omega)\right)^{\prime}\right) \cap$ $\left.L^{2}\left(0, T ; H_{\mathrm{D}}^{1}(\Omega)\right)\right)$, by interpolation its trace at zero is well-defined as an $L^{2}$ function (see e.g. [27]).

We study existence and uniqueness of weak solutions and possible higher regularity away from the singular set in certain weighted Sobolev spaces, motivated by analogous results for elliptic equations.

\section{Weighted Sobolev Spaces}

In this section, we discuss Sobolev spaces weighted by powers of the distance to the singular set. These spaces were use by Kondratiev [30] and other authors (in particular, Mazya and Plamenevskii [38]) to study elliptic equations on domains with conical points.

We begin by introducing the weight function, which is essentially the distance to the vertices.

$$
\rho(x)=\prod_{Q \in \mathcal{V}} d(x, Q) .
$$


where $d(x, Q)$ represents the (Euclidean) distance between the point $x$ and the vertex $Q$.

Given $m \in \mathbb{Z}_{+}$and $a \in \mathbb{R}$, we define the $m$ th-order weighted Sobolev space with weight $a$ :

$$
\mathcal{K}_{a}^{m}(\Omega)=\left\{u \in L_{l o c}^{2}(\Omega), \rho^{|\alpha|-a} \partial^{\alpha} u \in L^{2}(\Omega),|\alpha| \leq m\right\} .
$$

$\mathcal{K}_{a}^{m}$ is a Banach space with norm

$$
\|u\|_{\mathcal{K}_{a}^{m}(\Omega)}^{2}=\sum_{|\alpha| \leq m}\left\|\rho^{|\alpha|-a} \partial^{\alpha} u\right\|_{L^{2}(\Omega)}^{2}
$$

By definition $\mathcal{K}_{0}^{0}(\Omega)=L^{2}(\Omega)$. Furthermore, the spaces $\mathcal{K}_{a}^{m}$ form a scale, in the sense that:

$$
\mathcal{K}_{a}^{m} \subset \mathcal{K}_{a^{\prime}}^{m^{\prime}}, \quad m^{\prime} \leq m, a^{\prime} \leq a
$$

Whenever needed (e.g. when one must take derivatives of the weight function), we will implicitly replace $\rho$ with a smooth function $r_{\Omega}$, which is comparable to $\rho$ and induces an equivalent norm.

We define $\mathcal{K}_{a}^{-m}(\Omega), m \in \mathbb{Z}_{+}$by duality. Let $\dot{\mathcal{K}}_{a}^{m}(\Omega)$ denote the closure of $C_{c}^{\infty}(\Omega)$ in $\mathcal{K}_{a}^{m}(\Omega)$. Then, $\mathcal{K}_{-a}^{-m}=\left(\mathcal{K}_{m}^{a}(\Omega)\right)^{\prime}$. We can also define spaces on the boundary with good trace properties as follows. $\mathcal{K}_{a}^{m}(\partial \Omega)$ is the direct sum of the corresponding spaces on each of the edges with no compatibility at the vertices. The spaces $\mathcal{K}_{a}^{s}(\partial \Omega), s>0$, can then be defined as interpolation spaces. (We refer to [39] for a more in depth discussion of the spaces $\mathcal{K}_{a}^{m}$ and their properties.)

By a weighted form of Poincare's inequality (see e.g. [32, Lemma 3.5] for a proof),

$$
\|w\|_{\mathcal{K}_{1}^{0}(\Omega)}^{2} \leq C_{\Omega} \int_{\Omega}|\nabla w(x)|^{2} \mathrm{~d} x
$$

for any $w \in H_{\mathrm{D}}^{1}(\Omega)$, if no Neumann-Neumann vertices exist. Hence in this case,

$$
H_{\mathrm{D}}^{1}(\Omega) \subset \mathcal{K}_{1}^{1}(\Omega)
$$

We note that, if $u$ is continuous and $u \in \mathcal{K}_{1}^{1}(\Omega)$, then $u$ must vanish at the vertices of $\Omega$. By analogy, for general weights we set:

$$
\mathcal{H}_{a}:=\left\{w \in \mathcal{K}_{1+a}^{1}(\Omega), u=0 \text { on } \partial_{\mathrm{D}} \Omega\right\}
$$

We now introduce weighted spaces adapted to the transmission problem (3). Given the partition (1) of the domain $\Omega$, we define broken weighted Sobolev spaces [2,9] by

$$
\hat{\mathcal{K}}_{a}^{m}(\Omega):=\left\{w \in L^{2}(\Omega), w \in \mathcal{K}_{a}^{m}\left(\Omega_{k}\right), k=1,2, \ldots, K\right\}
$$


with the induced norm:

$$
\|w\|_{\hat{\mathcal{K}}_{a}^{m}(\Omega)}=\|w\|_{L^{2}(\Omega)}^{2}+\sum_{k=1}^{K}\|w\|_{\mathcal{K}_{a}^{m}\left(\Omega_{k}\right)} .
$$

The $L^{2}$ norm is redundant for positive weights.

We will analyze the IBVTP (3) by relating it to the corresponding elliptic boundaryvalue-transmission problem (BVTP for short):

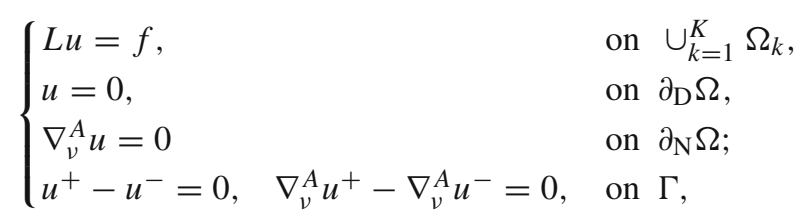

with the associated variational formulation:

$$
B[u, w]=\langle f, w\rangle, \quad \forall w \in H_{\mathrm{D}}^{1}(\Omega),
$$

for $u \in H_{\mathrm{D}}^{1}(\Omega)$. If $\mathcal{V}_{2}=\emptyset$, it follows from (8) that the bilinear form $B$ is strictly coercive on $\mathcal{H}_{0}$ and the solution operator of (10) is Fredholm of index zero. Using properties of the index, in particular, its behavior under conjugation with the weight, one readily obtains well-posedness of (10) in $\mathcal{H}_{a}$ for $a$ small enough.

When $\mathcal{V}_{2} \neq \varnothing$, the spectral properties of the solution operator change, since solutions are allowed to be locally constant near each vertex in $\mathcal{V}_{2}$. Solvability is restored by augmenting the space $\mathcal{H}_{0}$ with a finite dimensional space $W_{\mathrm{s}}$. A basis for this space consists of functions $\chi_{Q} \in C_{0}^{\infty}(\Omega)$, one for each $Q \in \mathcal{V}_{2}$, where $\chi_{Q}$ is constant equal to 1 in a neighborhood of $Q$. Higher regularity in $\hat{\mathcal{K}}_{1+a}^{m}$ can then be bootstrapped for more regular data.

To state the main well-posedness result for (10), we recall the following notation from [32]. For $m \in \mathbb{Z}_{+}$and $a \geq 0$, we set:

$$
\begin{array}{r}
V_{a+1}^{m+1}(\Omega)=\left\{u \mid u=u_{\mathrm{reg}}+w_{\mathrm{s}}, u_{\mathrm{reg}} \in \hat{\mathcal{K}}_{a+1}^{m+1}(\Omega) \cap \mathcal{K}_{a+1}^{1}(\Omega), w_{\mathrm{s}} \in \mathcal{W}_{\mathrm{s}}\right\} \\
\cap\left\{u \mid u=0 \text { on } \partial_{\mathrm{D}} \Omega, \nabla_{v}^{A} u=0 \text { on } \partial_{\mathrm{N}} \Omega, \nabla_{v}^{A} u^{+}=\nabla_{v}^{A} u^{-} \text {on } \Gamma\right\}
\end{array}
$$

We let $P: u \mapsto f$ denote the forward operator for Problem (10).

Theorem 1 ([32]) Let $m \in \mathbb{N}_{+}$. There exists $\eta=\eta(\Omega, L)>0$ such that, if $a \in$ $(0, \eta)$ and $f \in \hat{\mathcal{K}}_{a-1}^{m-1}(\Omega)$, then the elliptic problem (10) has a unique weak solution $u \in H_{\mathrm{D}}^{1}(\Omega)$, which can be uniquely decomposed as $u=u_{\mathrm{reg}}+w_{\mathrm{s}}$, where $u_{\mathrm{reg}} \in$ $\hat{\mathcal{K}}_{a+1}^{m+1}(\Omega) \cap \mathcal{H}_{a}(\Omega)$, and $w_{\mathrm{s}} \in \mathcal{W}_{\mathrm{s}}$.

Furthermore, the operator $P: u \rightarrow f$ gives an isomorphism between the space $V_{a+1}^{m+1}(\Omega)$ and $\hat{\mathcal{K}}_{a-1}^{m-1}(\Omega)$, and the following estimate holds: 


$$
\left\|u_{\mathrm{reg}}\right\|_{\mathcal{K}_{a+1}^{1}(\Omega)}+\left\|u_{\mathrm{reg}}\right\|_{\hat{\mathcal{K}}_{a+1}^{m+1}(\Omega)}+\left\|w_{\mathrm{s}}\right\|_{L^{2}(\Omega)} \leq C\|f\|_{\hat{\mathcal{K}}_{a-1}^{m-1}(\Omega)},
$$

with $C$ a constant that depends on $L$ and $\Omega$.

The constant $\eta$ is related to the singular exponents for the problem. We will refer to it as the singularity constant. We call $u_{\text {reg }}$ the regular part of $u$, as it belongs to the weighted Sobolev space $\hat{\mathcal{K}}_{a+1}^{m+1}(\Omega)$ and vanishes at the vertices, while we refer to $w_{\mathrm{s}}$ as the singular part of $u$, because it does not. Since $W_{\mathrm{s}}$ is a finite-dimensional space, we can replace the $L^{2}$ norm with any finite norm. We observe that when $\mathcal{V}_{2}=\emptyset$, i.e., all the vertices in $\mathcal{V}$ have at least one Dirichlet side and there is no interior vertex on

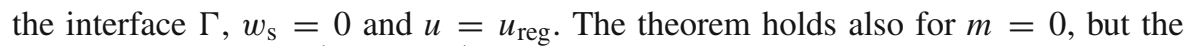
isomorphism is $\left.P: V_{1+a}^{1} \rightarrow\left(V_{1+a}^{1}\right)^{\prime}\right)$.

\section{Well-Posedness for the Parabolic Transmission Problem: Weak Solutions}

We begin our analysis of Problem (3) by temporarily assuming that $\mathcal{V}_{2}=\emptyset$. Our first result is an existence and uniqueness result for weak solutions in weighted spaces.

Theorem 2 Let $\eta$ be the singularity constant in the Theorem 1 and let $\mathcal{V}_{2}=\emptyset$. Then there exists a constant $0<\delta \leq \eta$, such that if $f \in L^{2}\left(0, T ;\left(V_{a+1}^{1}(\Omega)\right)^{\prime}\right)$ and if $g \in \mathcal{K}_{a}^{0}(\Omega)$ for $a \in(0, \delta)$, then there is a unique weak solution to (3) $u \in L^{2}\left(0, T ; \mathcal{K}_{a+1}^{1}(\Omega)\right) \cap H^{1}\left(0, T ;\left(V_{a+1}^{1}(\Omega)\right)^{\prime}\right)$ satisfying

$$
\|u\|_{L^{2}\left(0, T ; \mathcal{K}_{a+1}^{1}(\Omega)\right)}^{2}+\left\|u_{t}\right\|_{L^{2}\left(0, T ;\left(\mathcal{V}_{a+1}^{1}(\Omega)\right)^{\prime}\right)}^{2} \leq\|f\|_{L^{2}\left(0, T ;\left(\mathcal{V}_{a+1}^{1}(\Omega)\right)^{\prime}\right)}^{2}+\|g\|_{\mathcal{K}_{a}^{0}(\Omega)}^{2}
$$

A more precise estimate of $\delta$ will be presented later.

The theorem is a regularity theorem for weak solutions. Hence, the gist of the proof consists in analyzing the behavior of the solution at the singular points. The other steps in the proof generally follow a well-known approach (see e.g. the monographs [27] and [36]).

Proof We have

$$
f \in L^{2}\left(0, T ;\left(V_{a+1}^{1}(\Omega)\right)^{\prime}\right) \subset L^{2}\left(0, T ;\left(H_{\mathrm{D}}^{1}(\Omega)\right)^{\prime}\right), g \in \mathcal{K}_{a}^{0}(\Omega) \subset L^{2}(\Omega) .
$$

Hence, a standard Galerkin approximation gives the existence and uniqueness of a weak solution in $u \in L^{2}\left(0, T ; H_{\mathrm{D}}^{1}(\Omega)\right.$ ) to Problem (3) (see e.g. [27]).

To analyze the behavior in three main steps:

Step 1 We seek an adapted weight function $\vartheta(x)>0$ on $\Omega$, such that:

(a) $\vartheta$ behaves like $\rho$ pointwise, i.e., there exist two constants $0<C_{1}<C_{2}$ depending only on $\Omega$ and the coefficient matrix $A$, such that $C_{1} \rho<\vartheta<C_{2} \rho$ pointwise. 
(b) $\quad \vartheta$ is sufficiently smooth (at least $C^{1}$ ) inside each subdomain, except near the corners;

(c) $\quad \vartheta$ is continuous across the interface. that is, $\vartheta^{+}-\vartheta^{-}=0$ on $\Gamma$;

(d) $\quad \nabla_{v}^{A}\left(\vartheta^{a}\right)=0$ on $\partial_{\mathrm{N}} \Omega$ and $\nabla_{v}^{A}\left(\vartheta^{a}\right)^{+}=\nabla_{v}^{A}\left(\vartheta^{a}\right)^{-}=0$ on $\Gamma$.

We first construct $\vartheta$ in the case that the coefficient matrix $A=\left[a_{i j}\right]$ of the operator $L$ is constant on each subdomain. We denote a point in $\mathbb{R}^{2}$ by $(x, y)$. For each vertex $Q_{i}=\left(x_{i}, y_{i}\right), i=1, \ldots, N$ (where $N$ is the cardinality of $\mathcal{V})$, we denote by $\Omega_{i l}, l=1, \ldots, K(i)$, the subdomains of $\Omega$ that contain the vertex $Q_{i}$, where $K(i) \leq K$. Then we let

$\vartheta_{i}(x, y)=\vartheta_{i l}(x, y)=C_{i l}\left\{\left(x-x_{i}, y-y_{i}\right) A^{-1}\left(x-x_{i}, y-y_{i}\right)^{T}\right\}^{1 / 2}$,

defined in a neighborhood of the vertex $Q_{i}$ in each $\Omega_{i l}$. The constants $C_{i l}$ are chosen to make $\vartheta_{i}$ continuous across $\Gamma$, and we can set $C_{i 1}=1$ without loss of generality. We choose a constant $C_{0}>0$ small enough so that the sets

$$
\mathcal{B}_{Q_{i}}\left(C_{0}\right)=\left\{(x, y) \mid \vartheta_{i}(x, y) \leq C_{0}\right\}
$$

for each $Q_{i} \in \mathcal{V}$ are pairwise disjoint in $\Omega$. We next pick $\zeta_{i}$ to be a continuous function defined on $\left\{(x, y) \mid C_{0} / 2 \leq \vartheta_{i}(x, y) \leq C_{0}\right\}$ with the following properties:

(i) $\quad \zeta_{i}=\kappa\left(\vartheta_{i}\right)$ for some continuous function $\kappa$ defined on the closed interval $\left[C_{0} / 2, C_{0}\right]$

(ii) $\quad \kappa=1$ on $\left[C_{0} / 2,2 C_{0} / 3\right], \kappa=0$ on $\left[5 C_{0} / 6, C_{0}\right]$, and $\kappa$ is regular $\left(C^{1}\right.$ suffices) and non-increasing on $\left[C_{0} / 2, C_{0}\right]$.

We can now construct $\vartheta$ as follows. For notational convenience, we denote with $\vartheta_{0}$ the constant function 1 on $\bar{\Omega}$. Then, we let

$$
\vartheta(x, y)=\vartheta_{0}(x, y)=1
$$

if $(x, y) \in \Omega \backslash \bigcup_{Q_{i} \in \mathcal{V}} \mathcal{B}_{Q_{i}}\left(C_{0}\right)$; and

$$
\vartheta(x, y)=\vartheta_{i}(x, y)
$$

if $(x, y) \in \mathcal{B}_{Q_{i}}\left(C_{0} / 2\right)$; finally

$$
\vartheta(x, y)=\left(1-\zeta_{i}(x, y)\right) \vartheta_{0}(x, y)+\zeta_{i}(x, y) \vartheta_{i}(x, y)
$$

if $(x, y) \in \mathcal{B}_{Q_{i}}\left(C_{0}\right)$ but $(x, y) \notin \mathcal{B}_{Q_{i}}\left(C_{0} / 2\right)$. It is straightforward to check that $\vartheta$ satisfied conditions $1-4$ (note that since $\vartheta_{0}$ is constant, it satisfies the transmission conditions automatically irrespective of the values of $A$ ).

We now modify this construction for the case that $A$ is variable and regular on each subdomain. We just sketch the construction for brevity. In the constantcoefficient case, $\vartheta$ is given by (15c), that is, near each vertex $Q_{i}$ it is defined 
on sectors emanating from the the vertex $Q_{i}$ determined by the values of $A$. When $A$ is not constant on each subdomains, these sectors become deformed. However, sufficiently close to the vertex, they are still approximately straight sectors so that we can follow the same construction as in the constant case for a small neighborhood of each vertex $Q_{i}$, and then using again the functions $\zeta_{i}$ and $\vartheta_{0}$ construct $\vartheta$ globally in $\Omega$.

Step 2 We conjugate the operator $L$ with $\vartheta^{a}$. We let $\bar{u}=u / \vartheta^{a}$. If $u$ solves (3), then $\bar{u}$ solves the following problem:

$$
\begin{cases}\vartheta^{-a} L \vartheta^{a} \bar{u}+\bar{u}_{t}=\bar{f} & \text { in } \cup_{k=1}^{K} \Omega_{k} \times(0, T), \\ \bar{u}=0 & \text { on } \partial_{\mathrm{D}} \Omega \times(0, T), \\ \nabla_{v}^{A} \bar{u}=0 & \text { on } \partial_{\mathrm{N}} \Omega \times(0, T), \\ \bar{u}=\bar{g} & \text { in } \Omega \times\{t=0\}, \\ \bar{u}^{+}=\bar{u}^{-}, \quad \nabla_{v}^{A} \bar{u}^{+}=\nabla_{v}^{A} \bar{u}^{-} & \text {on } \Gamma \times(0, T),\end{cases}
$$

taking into accounts the conditions 4-4 satisfied by $\vartheta$, where $\bar{f}=f / \vartheta^{a} \in$ $L^{2}\left(0, T ; \mathcal{K}_{-1}^{-1}(\Omega)\right) \subset L^{2}\left(0, T ;\left(H_{\mathrm{D}}^{1}(\Omega)\right)^{\prime}\right), \bar{g}=g / \vartheta^{a} \in L^{2}(\Omega)$. We solve Problem (16) via a Galerkin approximation. We observe that the transmission conditions and the Neumann condition are not those naturally associated to the conjugated operator, so we will need to construct an adapted Garlerkin basis in 4.

We begin by noticing that a weak solution $\bar{u} \in L^{2}\left(0, T ; H_{\mathrm{D}}^{1}(\Omega)\right)$ must satisfy for all $v \in H_{\mathrm{D}}^{1}(\Omega)$ :

$$
\begin{aligned}
& \left\langle f-\bar{u}_{t}, v\right\rangle=\left\langle\vartheta^{-a} L \vartheta^{a} \bar{u}, v\right\rangle=(\nabla \bar{u}, \nabla v)_{A}-a^{2}\left(\frac{\bar{u} v}{\vartheta^{2}} \nabla \vartheta, \nabla \vartheta\right)_{A} \\
& +a\left(\frac{\bar{u}}{\vartheta} \nabla v, \nabla \vartheta\right)_{A}-a\left(\frac{v}{\vartheta} \nabla \bar{u}, \nabla \vartheta\right)_{A}=: B_{a}[\bar{u}, v]
\end{aligned}
$$

where we used the notation $(X, Y)_{A}:=\int_{\Omega} X^{T} A Y \mathrm{~d} x$. We view (17) as an elliptic problem.

We show next that $B_{a}$ is continuous and coercive on $H_{\mathrm{D}}^{1}(\Omega)$. Continuity follows from the weighted form of Poincaré's inequality (8) and Condition 4 for $\vartheta$ :

$$
\begin{gathered}
B_{a}[\bar{u}, v] \leq \alpha_{1}\|\nabla \bar{u}\|_{L^{2}(\Omega)}\|\nabla v\|_{L^{2}(\Omega)}+\alpha_{2}\|\bar{u} / \vartheta\|_{L^{2}(\Omega)}\|v / \vartheta\|_{L^{2}(\Omega)}+ \\
\alpha_{3}\|\bar{u} / \vartheta\|_{L^{2}(\Omega)}\|\nabla v\|_{L^{2}(\Omega)}+\alpha_{4}\|\nabla \bar{u}\|_{L^{2}(\Omega)}\|v / \vartheta\|_{L^{2}(\Omega)} \\
\leq \alpha\|\nabla \bar{u}\|_{L^{2}(\Omega)}\|\nabla v\|_{L^{2}(\Omega)}
\end{gathered}
$$

for some positive constant $\alpha=\alpha(a)>0$. To ensure coercivity of the bilinear form, we need to restrict the range of the weight $a$. Since $A$ is bounded above and below, it is enough to find a lower bound for $B_{a}$, assuming $A$ is constant. 
In this case, we have an explicit formula for $\vartheta$, formula (15c). We then have:

$$
\begin{aligned}
B_{a}[\bar{u}, \bar{u}]= & (\nabla \bar{u}, \nabla \bar{u})_{A}-a^{2}\left(\frac{\bar{u}^{2}}{\vartheta^{2}} \nabla \vartheta, \nabla \vartheta\right)_{A} \\
\geq & \bigcup_{Q_{i} \in \mathcal{V}} \int_{\mathcal{B}_{Q_{i}}\left(C_{0} / 2\right)}(\nabla \bar{u})^{T} A(\nabla \bar{u}) \mathrm{d} S \\
& \left.-a^{2} \int_{\mathcal{B}_{Q_{i}}\left(C_{0} / 2\right)} \frac{\bar{u}^{2}}{\left(x-x_{i}, y-y_{i}\right)^{T} A^{-1}\left(x-x_{i}, y-y_{i}\right)} \mathrm{d} S\right) \\
& \left.+\beta_{0}\|\nabla \bar{u}\|_{L^{2}\left(\Omega \backslash \cup_{Q_{i} \in \mathcal{V}}^{2} \mathcal{B}_{Q_{i}}\left(C_{0} / 2\right)\right)}\right) \\
& -\gamma_{0} a^{2}\|\bar{u}\|_{L^{2}\left(\Omega \backslash \cup_{Q_{i} \in \mathcal{V}} \mathcal{B}_{Q_{i}}\left(C_{0} / 2\right)\right)}
\end{aligned}
$$

for some $\beta_{0}, \gamma_{0}>0$ that doe not depend on $\bar{u}$. We focus on the first term of (19). For each vertex $Q_{i}$ we define $r_{i}=\inf _{\vartheta(X)=C_{0} / 2} d\left(X, Q_{i}\right)$ so that the part of the disk centered at $Q_{i}$ in $\Omega$ with radius $r_{i}$ lies inside $\mathcal{B}_{Q_{i}}\left(C_{0} / 2\right)$. We denote this sector by $D_{i}$ and its aperture angle by $\omega_{i}$. Since $A$ is symmetric positive definite, by the weighted Poincaré's inequality (8) it follows that

$$
\begin{gathered}
\int_{\mathcal{B}_{Q_{i}}\left(C_{0} / 2\right)}\left(\bar{u}_{x}, \bar{u}_{y}\right)^{T} A\left(\bar{u}_{x}, \bar{u}_{y}\right) \mathrm{d} S \geq \int_{D_{i}}\left(\bar{u}_{x}, \bar{u}_{y}\right)^{T} A\left(\bar{u}_{x}, \bar{u}_{y}\right) \mathrm{d} S \geq \beta_{1}\|\nabla \bar{u}\|_{L^{2}\left(D_{i}\right)}^{2} \\
\quad \geq \beta_{1}\left(\frac{\pi}{2 \omega_{i}}\right)^{2} \int_{D_{i}}\left(\frac{\bar{u}}{d\left((x, y), Q_{i}\right)}\right)^{2} \mathrm{~d} S \geq \frac{\beta_{2}}{\omega_{i}^{2}} \int_{D_{i}} \frac{\bar{u}^{2}}{\left(x-x_{i}, y-y_{i}\right)^{T} A^{-1}\left(x-x_{i}, y-y_{i}\right)} \mathrm{d} S \\
\quad \geq \frac{\beta_{2}}{\omega_{i}^{2}} \int_{\mathcal{B}_{Q_{i}}\left(C_{0} / 2\right)} \frac{\bar{u}^{2}}{\left(x-x_{i}, y-y_{i}\right)^{T} A^{-1}\left(x-x_{i}, y-y_{i}\right)} \mathrm{d} S-\frac{\beta_{3}}{\omega_{i}^{2} r_{i}^{2}}\|\bar{u}\|_{L^{2}\left(\mathcal{B}_{Q_{i}}\left(C_{0} / 2\right)\right)}^{2}
\end{gathered}
$$

for some constant $\beta_{2}, \beta_{3}>0$ depending only on $A$. We then let $\omega=\max _{Q_{i} \in \mathcal{V}}\left\{\omega_{i}\right\}$ and choose $\delta=\min \left\{\frac{\sqrt{\beta_{2}}}{\omega}, \eta\right\}$. By combining the inequalities (19) and (20) we can conclude that $a^{2}<\beta_{2} / \omega_{i}^{2}$ for each vertex $Q_{i}$ if $0<a<\delta$. Consequently, if $a$ lies in this range, then there exist constants $\beta, \gamma>0$ such that

$$
B_{a}[\bar{u}, \bar{u}] \geq \beta\|\nabla \bar{u}\|_{L^{2}(\Omega)}^{2}-\gamma\|\bar{u}\|_{L^{2}(\Omega)}^{2} .
$$

for all $\bar{u} \in H_{\mathrm{D}}^{1}(\Omega)$.

Step 3 We construct an orthonormal basis of $L^{2}(\Omega)$, which is also orthogonal in $H_{\mathrm{D}}^{1}(\Omega)$, adapted to the boundary and transmission conditions for Problem 16. Recall that we assume here that $\mathcal{V}_{2}=\emptyset$. Then, $H_{\mathrm{D}}^{1}(\Omega) \subset \mathcal{K}_{1}^{1}(\Omega)$. According to Theorem 1, the solution operator of the BVTP $(10)$ maps $L^{2}(\Omega) \subset \mathcal{K}_{a-1}^{0}(\Omega)$ into $V_{1+a}^{2} \subset L^{2}(\Omega)$ for $0>a>\eta$ boundedly. Hence, by Rellich Theorem it is a compact operator on $L^{2}(\Omega)$ and self adjoint, since $A$ is symmetric. 
Consequently, there exists a countable orthonormal basis of $L^{2}(\Omega)$ consisting of eigenfunctions of $L,\left\{w_{i}\right\}_{i=1}^{\infty}$. But $w_{i} \in V_{1+a}^{2}$, so it satisfies the boundary and transmission conditions in (16). Hence by Green's formulas:

$$
B\left[w_{i}, w_{j}\right]=\lambda_{i}\left\langle w_{i}, w_{j}\right\rangle=\lambda_{j}\left\langle w_{i}, w_{j}\right\rangle=0
$$

as $\lambda_{i} \neq \lambda_{j}$ (if $\lambda_{i}=\lambda_{j}$, we use that the basis in orthogonal with respect to the $L^{2}$ inner product); that is, $\left\{w_{i}\right\}_{i=1}^{\infty}$ is also a complete B-orthogonal basis in $H_{\mathrm{D}}^{1}(\Omega)$. We take $\left\{w_{i}\right\}_{i=1}^{\infty}$ as the basis of the Galerkin approximation for the parabolic problem (16). For $m \in \mathbb{N}$, we define

$$
\bar{u}_{m}(t, \cdot)=\sum_{i=1}^{m} d_{m}^{i}(t) w_{i}, \quad i=1,2, \ldots, m
$$

and denote $\bar{u}_{m}(t):=\bar{u}_{m}(t, \cdot)$. The function $\bar{u}_{m}$ is an exact solution of the variational problem (6) in the span of $w_{1}, \ldots, w_{m}$ if $\left\{d_{m}^{i}(t)\right\}_{i=1}^{m}$ satisfies the following system of ODEs:

$$
B_{a}\left[\bar{u}_{m}(t), w_{i}\right]+\left(\bar{u}_{m}^{\prime}(t), w_{i}\right)=\left\langle\bar{f}, w_{i}\right\rangle, \quad i=1,2, \ldots, m,
$$

with initial conditions

$$
d_{m}^{i}(0)=\left(\bar{g}, w_{i}\right), \quad i=1,2, \ldots, m,
$$

where $\bar{f}=f / \vartheta^{a}, \bar{g}=g / \vartheta^{a}$. Given the properties of $B_{a}$, there exists a unique solution for each $m \in \mathbb{N}$. In addition, a simple energy estimate, using the coercivity and boundedness of $B_{a}$, gives

$$
\begin{array}{r}
\left\|\bar{u}_{m}\right\|_{L^{2}\left(0, T ; H_{\mathrm{D}}^{1}(\Omega)\right)}^{2}+\left\|\bar{u}_{m}^{\prime}\right\|_{L^{2}\left(0, T ; H^{-1}(\Omega)\right)}^{2}+\left\|\bar{u}_{m}\right\|_{L^{\infty}\left(0, T ; L^{2}(\Omega)\right)}^{2} \\
\leq C\left(\|\bar{f}\|_{L^{2}\left(0, T ;\left(H_{\mathrm{D}}^{1}(\Omega)\right)^{\prime}\right)}^{2}+\|\bar{g}\|_{L^{2}(\Omega)}^{2}\right),
\end{array}
$$

with the constant $C$ depending only on $\Omega$ and the coefficient matrix $A$. Let $u_{m}=\vartheta^{a} \bar{u}_{m}$. For each $t>0$, we have from the weighted Poincaré's estimate (8) that

$$
\left\|\frac{u_{m}}{\vartheta^{a+1}}\right\|_{L^{2}(\Omega)}=\left\|\frac{\bar{u}_{m}}{\vartheta}\right\|_{L^{2}(\Omega)} \leq C\left\|\nabla \bar{u}_{m}\right\|_{L^{2}(\Omega)},
$$

and

$\left\|\frac{\nabla u_{m}}{\vartheta^{a}}\right\|_{L^{2}(\Omega)} \leq C\left(\left\|\nabla \frac{u_{m}}{\vartheta^{a}}\right\|_{L^{2}(\Omega)}+\left\|\frac{u_{m}}{\vartheta^{a+1}}\right\|_{L^{2}(\Omega)}\right) \leq C\left\|\nabla \bar{u}_{m}\right\|_{L^{2}(\Omega)}$.

It follows from (23)-(24) that $u_{m}$ is uniformly bounded $L^{2}\left(0, T ; \mathcal{K}_{a+1}^{1}(\Omega)\right)$. Consequently, there exists a subsequence (not relabeled) that converges weakly 
to some element $U$ of $L^{2}\left(0, T ; \mathcal{K}_{a+1}^{1}(\Omega)\right)$. However, since $\bar{u}_{m}$ satisfies (22) and the Galerkin basis is complete in $H_{\mathrm{D}}^{1}(\Omega)$, it is straightforward to check that $\bar{U}=\vartheta^{-a} U$ satisfies (17), from which it follows immediately that $U$ satisfies (4) and, hence, must agree with $u$. Then by (24) $u$ satisfies

$$
\|u\|_{L^{2}\left(0, T ; \mathcal{K}_{a+1}^{1}(\Omega)\right)}^{2} \leq\|f\|_{L^{2}\left(0, T ;\left(V_{a+1}^{1}(\Omega)\right)^{\prime}\right)}^{2}+\|g\|_{\mathcal{K}_{a}^{0}(\Omega)}^{2} .
$$

Similarly, passing to the limit $m \rightarrow \infty$ in (23) gives

$$
\|u\|_{L^{\infty}\left(0, T ; \mathcal{K}_{a}^{0}(\Omega)\right)}^{2}+\left\|u_{t}\right\|_{L^{2}\left(0, T ;\left(V_{a+1}^{1}(\Omega)\right)^{\prime}\right)}^{2} \leq\|f\|_{L^{2}\left(0, T ;\left(V_{a+1}^{1}(\Omega)\right)^{\prime}\right)}^{2}+\|g\|_{\mathcal{K}_{a}^{0}(\Omega)}^{2} .
$$

The proof of the theorem is now complete.

We next improve the regularity in time of the weak solution, if the data $f$ and $g$ are more regular. With Theorem 2 at hand, bootstrap of regularity follows in a manner similar to that for smooth domains.

Corollary 1 Let $u$ be the weak solution of (3) given in Theorem 2. If $f \in$ $L^{2}\left(0, T ; \mathcal{K}_{a}^{0}(\Omega)\right)$ and $g \in \mathcal{K}_{a+1}^{1}(\Omega) \cap H_{\mathrm{D}}^{1}(\Omega)$ with $a \in(0, \delta)$, then $u \in$ $L^{\infty}\left(0, T ; \mathcal{K}_{a+1}^{1}(\Omega)\right) \cap H^{1}\left(0, T ; \mathcal{K}_{a}^{0}(\Omega)\right)$.

Proof We utilize the notation introduced in the proof of Theorem 2. From the proof of Theorem 2, $\bar{u}$ is a limit of approximate solutions $\bar{u}_{m}$, where $\bar{u}_{m}$ solves Problem (16), and estimate (23) holds. Then $\bar{f} \in L^{2}\left(0, T ; L^{2}(\Omega)\right.$, and $\bar{g} \in L^{2}\left(0, T ; H_{\mathrm{D}}^{1}(\Omega)\right)$. By the coercivity and boundedness of the bilinear form $B$ on $H_{\mathrm{D}}^{1}(\Omega)$, it follows that for each $0<t<T$,

$$
\begin{aligned}
\left\|\bar{u}_{m}(t)\right\|_{H_{\mathrm{D}}^{1}(\Omega)}^{2} \leq & \beta_{4} B\left[\bar{u}_{m}(t), \bar{u}_{m}(t)\right] \leq \beta_{4}\left(B\left[\bar{u}_{m}(0), \bar{u}_{m}(0)\right]\right. \\
& \left.+\int_{0}^{t} B\left[\bar{u}_{m}(\tau), \bar{u}_{m}^{\prime}(\tau)\right] \mathrm{d} \tau\right) \\
\leq & \beta_{4} B\left[\bar{u}_{m}(0), \bar{u}_{m}(0)\right]+\beta_{4} \int_{0}^{t}-\left(\bar{u}_{m}^{\prime}(\tau), \bar{u}_{m}^{\prime}(\tau)\right)+\left(\bar{u}_{m}^{\prime}(\tau), \bar{f}(\tau)\right) \mathrm{d} \tau \\
\leq & \beta_{5}\|\bar{g}\|_{H_{\mathrm{D}}^{1}(\Omega)}^{2}+\beta_{6}\|\bar{f}\|_{L^{2}\left(0, T ; L^{2}(\Omega)\right)}^{2}-\frac{\beta_{4}}{2}\left\|\bar{u}_{m}^{\prime}\right\|_{L^{2}\left(0, T ; L^{2}(\Omega)\right)}^{2},
\end{aligned}
$$

where we used (22) in the integral. Recalling that $u_{m}=\vartheta^{a} \bar{u}^{m}$, we have that

$$
\left\|u_{m}\right\|_{L^{\infty}\left(0, T ; \mathcal{K}_{a+1}^{1}(\Omega)\right)}^{2}+\left\|u_{m}^{\prime}\right\|_{L^{2}\left(0, T ; \mathcal{K}_{a}^{0}(\Omega)\right)}^{2} \leq C\left(\|g\|_{\mathcal{K}_{a+1}^{1}(\Omega)}^{2}+\|\bar{f}\|_{L^{2}\left(0, T ; \mathcal{K}_{a}^{0}(\Omega)\right)}^{2}\right) .
$$

Passing to the limit $m \rightarrow \infty$ and using the weak lower semicontinuity of the norms then yields $u \in L^{\infty}\left(0, T ; \mathcal{K}_{a+1}^{1}(\Omega)\right) \cap L^{\infty}\left(0, T ; H_{\mathrm{D}}^{1}(\Omega)\right)$ and $u_{t} \in L^{2}\left(0, T ; \mathcal{K}_{a}^{0}(\Omega)\right)$, with the same bound as in $(25)$.

Remark 2 Using the above corollary, we can bootstrap space regularity once. First, we note that $u$ solves the parabolic problem (3) strongly, that is, $L u=f-u_{t}$ a.e. in 
$\cup_{k=1}^{K} \Omega_{k} \times T$. Since $f-u_{t} \in L^{2}\left(0, T ; \mathcal{K}_{a}^{0}(\Omega)\right)$, from elliptic regularity (Theorem 1$)$ we deduce $u=P^{-1}\left(f-u_{t}\right) \in L^{2}\left(0, T ; V_{b+1}^{2}(\Omega)\right)$ for any $0<b<\min \{\eta, a+1\}$, where $\eta$ is the singularity constant in Theorem 1 .

To bootstrap regularity in the space variables further, we need to impose compatibility conditions on the data. We measure higher space regularity in broken weighted spaces $\hat{\mathcal{K}}_{1+a}^{m}$.

Lemma 1 Let $\mathcal{V}_{2}=\emptyset$. Assume that $g \in V_{a+1}^{2}(\Omega), f_{t} \in L^{2}\left(0, T ;\left(V_{a+1}^{1}(\Omega)\right)^{\prime}\right), f(0)-$ $P g \in \mathcal{K}_{a}^{0}(\Omega)$, for $a \in(0, \delta)$. Let $u$ be the weak solution of IBVTP (3). Then, $u_{t}$ belongs to $L^{2}\left(0, T ; \mathcal{K}_{a+1}^{1}(\Omega)\right) \cap L^{2}\left(0, T ; H_{\mathrm{D}}^{1}(\Omega)\right)$ and is the unique weak solution of the IBVTP

$$
\begin{cases}L w+w_{t}=f_{t}, & \text { in } \cap_{k=1}^{K} \Omega_{k} \times(0, T), \\ w=0, & \text { on } \partial_{\mathrm{D}} \Omega \times(0, T), \\ \nabla_{v}^{A} w=0, & \text { on } \partial_{\mathrm{N}} \Omega \times(0, T), \\ w^{+}=w^{-}, \quad \nabla_{v}^{A} w^{+}=\nabla_{v}^{A} w^{-}, & \text {on } \Gamma \times(0, T), \\ w(0)=f(0)-P g, & \text { on } \Omega \times\{t=0\} .\end{cases}
$$

Proof By classical results, there exists a unique weak solution of (26), satisfying

$$
B[w, v]+\left\langle w_{t}, v\right\rangle=\left\langle f_{t}, v\right\rangle \quad \forall v \in H_{\mathrm{D}}^{1}(\Omega) \text { and a.e. } 0 \leq t \leq T,
$$

along with the initial condition $w(0)=f(0)-P g \in \mathcal{K}_{a}^{0}(\Omega)$ in trace sense. By Theorem $2 w \in L^{2}\left(0, T ; \mathcal{K}_{a+1}^{1}(\Omega)\right) \cap L^{2}\left(0, T ; H_{\mathrm{D}}^{1}(\Omega)\right) \cap L^{\infty}\left(0, T ; \mathcal{K}_{a}^{0}(\Omega)\right)$. Let

$$
\tilde{u}(t)=\int_{0}^{t} w(\tau) \mathrm{d} \tau+g
$$

for each $t \in[0, T]$. By interpolation, $w \in C\left([0, T] ; \mathcal{K}_{a}^{0}(\Omega)\right)$, hence the function $\tilde{u}(t)$ is well defined and is Lipschitz continuous in the $\mathcal{K}_{a}^{0}(\Omega)$ norm. In particular, $\left(\tilde{u}_{t}, v\right)=(w, v)$ for $t \in[0, T]$ pointwise. Integrating (27) gives

$$
\int_{0}^{t} B[w(\tau), v] d \tau+\int_{0}^{t}\left\langle w_{t}(\tau), v\right\rangle=\int_{0}^{t}\left\langle f_{t}(\tau), v\right\rangle \mathrm{d} \tau .
$$

Recall that $v$ is constant in time. Given that $w \in L^{2}\left(0, T ; \mathcal{K}_{a+1}^{1}(\Omega)\right), w_{t} \in$ $L^{2}\left(0, T ;\left(V_{a+1}^{1}(\Omega)\right)^{\prime}\right), f_{t} \in L^{2}\left(0, T ;\left(V_{a+1}^{1}(\Omega)\right)^{\prime}\right)$, we can conclude that

$$
B[\tilde{u}(t)-\tilde{u}(0), v]+\langle w(t)-w(0), v\rangle=\langle f(t)-f(0), v\rangle .
$$

As $g=\tilde{u}(0) \in V_{a+1}^{2}(\Omega)$, we can apply Green's formulas and get

$$
B[\tilde{u}(0), v]+\langle w(0)-\langle f(0), v\rangle=\langle L g+f(0)-L g-f(0), v\rangle=0
$$


so that (29) becomes

$$
B[\tilde{u}(t), v]+\left\langle\tilde{u}_{t}(t), v\right\rangle=(f(t), v) .
$$

By uniqueness of weak solutions, $\tilde{u}=u$. Hence, $\tilde{u}_{t}=w=u_{t}$ solves (26) and has the stated regularity.

As expected, we can achieve higher regularity by imposing more compatibility conditions on the data.

Theorem 3 Let $\delta>0$ be as in Theorem 2 and assume $\mathcal{V}_{2}=\emptyset$. Given $m \in \mathbb{N}$ and $a \in(0, \delta)$, assume $g \in \hat{\mathcal{K}}_{a+1}^{2 m+1}(\Omega)$ and

$$
\begin{array}{lr}
\frac{\mathrm{d}^{i} f}{\mathrm{~d} t^{i}} \in C\left([0, T] ; \hat{\mathcal{K}}_{a}^{2 m-1-2 i}(\Omega)\right), & i=0,1, \ldots, m-1, \\
\frac{\mathrm{d}^{i} f}{\mathrm{~d} t^{i}} \in L^{2}\left(0, T ; \hat{\mathcal{K}}_{a}^{2 m-2 i}(\Omega)\right), & i=0,1, \ldots, m .
\end{array}
$$

Suppose also the following mth-order compatibility conditions hold:

$$
\begin{aligned}
& g_{0}:=g \in V_{a+1}^{2}(\Omega) \cap \hat{\mathcal{K}}_{a+1}^{2 m+1}(\Omega), \\
& g_{1}:=f(0)-P g_{0} \in V_{a+1}^{2}(\Omega) \cap \hat{\mathcal{K}}_{a+1}^{2 m-1}(\Omega), \\
& \ldots \\
& g_{m-1}:=\frac{\mathrm{d}^{m-2} f}{\mathrm{~d} t^{m-2}}(0)-P g_{m-2} \in V_{a+1}^{2}(\Omega) \cap \hat{\mathcal{K}}_{a+1}^{3}(\Omega), \\
& g_{m}:=\frac{\mathrm{d}^{m-1} f}{\mathrm{~d} t^{m-1}}(0)-P g_{m-1} \in \mathcal{K}_{a+1}^{1}(\Omega) \cap H_{\mathrm{D}}^{1}(\Omega)
\end{aligned}
$$

If $u$ is the weak solution of Problem (3) on $[0, T]$, then

$$
\frac{\mathrm{d}^{i} u}{\mathrm{~d} t^{i}} \in L^{\infty}\left(0, T ; \hat{\mathcal{K}}_{a+1}^{2 m+1-2 i}(\Omega)\right) \cap L^{\infty}\left(0, T ; \mathcal{K}_{a+1}^{1}(\Omega)\right) \quad i=0,1, \ldots, m
$$

and the following estimate holds

$$
\begin{aligned}
\sum_{i=0}^{m}\left\|\frac{\mathrm{d}^{i} u}{\mathrm{~d} t^{i}}\right\|_{L^{\infty}\left(0, T ; \hat{\mathcal{K}}_{a+1}^{2 m+1-2 i}(\Omega)\right)} \leq & C\left(\sum_{i=0}^{m}\left\|\frac{\mathrm{d}^{i} f}{\mathrm{~d} t^{i}}\right\|_{L^{2}\left(0, T ; \hat{\mathcal{K}}_{a}^{2 m-2 i}(\Omega)\right)}\right. \\
& \left.+\sum_{i=0}^{m-1}\left\|\frac{\mathrm{d}^{i} f}{\mathrm{~d} t^{i}}\right\|_{L^{\infty}\left(0, T ; \hat{\mathcal{K}}_{a}^{2 m-1-2 i}(\Omega)\right)}+\sum_{i=0}^{m}\left\|g_{i}\right\|_{\hat{\mathcal{K}}_{a+1}^{2 m+1-2 i}(\Omega)}\right),
\end{aligned}
$$

with $C$ depending on $m, \Omega, T$ and the coefficients of $L$.

The proof uses induction on $m$ and Lemma 1. For brevity's sake, we omit the proof and refer to [53]. 
Remark 3 We give an example of data that satisfy the compatibility conditions (32). We recall that $w_{i}$ is an eigenfunction of the operator $L$ satisfying the boundary and transmission conditions. Then, Theorem 1 allows to bootstrap the regularity of the eigenfunctions and guarantees that $w_{j} \in V_{a+1}^{2} \cap \hat{\mathcal{K}}_{a+1}^{2 m+1}(\Omega)$ for all $m \in \mathbb{N}$. Next, we pick $f$ and $g$ to be finite linear combinations of the eigenfunctions:

$$
g=c_{1} w_{1}+c_{2} w_{2}+\cdots+c_{N} w_{N}
$$

and

$$
f=d_{1}(t) w_{1}+d_{2}(t) w_{2}+\cdots+d_{\mathrm{N}}(t) w_{\mathrm{N}},
$$

where $\left\{c_{l}\right\}_{l=1}^{N} \in \mathbb{R},\left\{d_{l}(t)\right\}_{l=1}^{N} \in C^{m}[0, T]$. Then the compatibility conditions automatically hold.

We now turn to the case when $\mathcal{V}_{2} \neq \emptyset$, which means that Neumann-Neumann vertices are present and the interface $\Gamma$ has singular points. We study well-posedness of the parabolic problem again by viewing it as an elliptic problem with time-dependent forcing and exploiting Theorem 1 . Higher regularity can be obtained by imposing more compatibility conditions on the data in the spirit of Theorem 3.

Theorem 4 Let $u$ be the unique weak solution of the parabolic problem (3) on [0,T]. Let $\eta$ be the singularity constant in Theorem 1 and assume that

$$
\begin{gathered}
f_{t t} \in L^{2}\left(0, T ;\left(H_{\mathrm{D}}^{1}(\Omega)\right)^{\prime}\right), \quad f_{t} \in C\left(0, T ; L^{2}(\Omega)\right), \quad f \in C\left(0, T ; \hat{\mathcal{K}}_{a}^{2}(\Omega)\right), \\
g \in V_{a+1}^{2}(\Omega), \quad g_{1}=f(0)-P g \in V_{a+1}^{2}(\Omega), \quad g_{2}=f_{t}(0)-P g_{1} \in L^{2}(\Omega) .
\end{gathered}
$$

Then, the weak solution $u$ of Problem 3 can be decomposed as $u=u_{\mathrm{reg}}+w_{\mathrm{s}}$, where

$$
u_{\text {reg }} \in W^{2, \infty}\left(0, T ; L^{2}(\Omega)\right) \cap W^{1, \infty}\left(0, T ; \hat{\mathcal{K}}_{a+1}^{2}(\Omega)\right) \cap L^{\infty}\left(0, T ; \hat{\mathcal{K}}_{a+1}^{4}(\Omega)\right) ;
$$

and $w_{\mathrm{s}} \in W_{\mathrm{s}}$. In addition, the following bound holds:

$$
\begin{aligned}
& \left\|w_{\mathrm{s}}\right\|_{L^{\infty}\left(0, T ; \hat{H}^{2}(\Omega)\right)}+\left\|u_{\mathrm{reg}}\right\|_{W^{2, \infty}\left(0, T ; L^{2}(\Omega)\right)}+\left\|u_{\mathrm{reg}}\right\|_{W^{1, \infty}\left(0, T ; \hat{\mathcal{K}}_{a+1}^{2}(\Omega)\right)} \\
& \quad+\left\|u_{\mathrm{reg}}\right\|_{L^{\infty}\left(0, T ; \hat{\mathcal{K}}_{a+1}^{4}(\Omega)\right)} \\
& \leq C\left(\left\|f_{t t}\right\|_{L^{2}\left(0, T ;\left(H_{\mathrm{D}}^{1}(\Omega)\right)^{\prime}\right)}+\left\|f_{t}\right\|_{C\left(0, T ; L^{2}(\Omega)\right)}+\|f\|_{C\left(0, T ; \hat{\mathcal{K}}_{a}^{2}(\Omega)\right)}+\|g\|_{V_{a+1}^{2}(\Omega)}\right. \\
& \left.\quad+\|f(0)-P g\|_{V_{a+1}^{2}(\Omega)}\right),
\end{aligned}
$$

with $C$ depending only on $\Omega, a$, and $L$.

Proof Existence and uniqueness of the weak solution follows exactly as in the case $\mathcal{V}_{2}=\emptyset$. Differentiating (3) twice gives that $u_{t t} \in L^{\infty}\left(0, T ; L^{2}(\Omega)\right)$ by the Galerkin approximation and energy estimates. Since $P u_{t}=f_{t}-u_{t t}$ for a.e. $t>0$, where $P$ is the solution operator for the elliptic problem (10), and $P$ gives an isomorphism 
from $V_{a+1}^{2}(\Omega)$ to $\mathcal{K}_{a-1}^{0}(\Omega) \supset L^{2}(\Omega)$, we obtain from (12) that $u_{t}=P^{-1}\left(f_{t}-\right.$ $\left.u_{t t}\right) \in L^{\infty}\left(0, T ; V_{a+1}^{2}(\Omega)\right)$. Similarly, $u=P^{-1}\left(f-u_{t}\right)$ implies a bound on the $L^{\infty}\left(0, T ; \hat{\mathcal{K}}_{a+1}^{4}\right)$ norm of $u$.

\section{Vertex Singularities}

In this section, we study more closely the singularities in the solution of Problem 10 at the vertices, in the case that the operator $L$ has piecewise-constant coefficients in each subdomain $\Omega_{k}, k=1, \ldots, K$, generalizing the results of Kondratiev [30] for the Laplace operator and of Nicaise $[45,46]$ for $L$ a scalar multiple of the Laplacian in each $\Omega_{k}$. We assume $\mathcal{V}_{2}=\emptyset$ in order to provide an explicit characterization of the first singular exponent, which is determined by the value of $\eta$. Being able to compute or estimate $\eta$ will be important for applications to the FEM in Sect. 6.

Theorem 5 Let $\eta>0$ be the singularity constant in Theorem 1 , and let $\delta>0$ be the constant in Theorem 2. Assume $\mathcal{V}_{2}=\emptyset$. Assume also the coefficient matrix A of the operator $L$ is constant on each subdomain $\Omega_{k}, k=1,2, \ldots, K$. Then

$$
\max \delta^{2}=\eta^{2}=\inf _{Q_{i} \in \mathcal{V}_{1}, \bar{u} \in H_{\mathrm{D}}^{1}(\Omega), \bar{u} \neq 0} \frac{\int_{\mathcal{B}_{i}\left(C_{0} / 2\right)}(\nabla \bar{u})^{T} A(\nabla \bar{u}) \mathrm{d} S}{\int_{\mathcal{B}_{Q_{i}}\left(C_{0} / 2\right)} \frac{\bar{u}^{2}}{\left(x-x_{i}, y-y_{i}\right) A^{-1}\left(x-x_{i}, y-y_{i}\right)^{T}} \mathrm{~d} S},
$$

where the sets $\mathcal{B}_{Q_{i}}$ are defined in (14) and $Q_{i}=\left(x_{i}, y_{i}\right)$.

Remark 4 It is known that the singular exponents can be computed by solving suitable eigenvalue problems after blowing up the vertex singularities. Then, formula (33) can be thought of as a Rayleigh quotient for these eigenvalue problems. In particular, if $L=-\triangle$ on $\Omega$, then we recover the well-known formula

$$
\max \delta=\eta=\min _{Q_{i} \in \mathcal{V}}\left\{\frac{\pi}{\sigma_{i} \omega_{i}}\right\}
$$

where $\omega_{i}$ is the interior angle of $\Omega$ in the vertex $Q_{i}, \sigma_{i}=1$ if $Q_{i}$ has two Dirichlet sides, $\sigma_{i}=2$ if $Q_{i}$ has one Neumann side and one Dirichlet side. (We refer to [53] for a proof of this fact.)

Proof We first prove that $\max \delta=\eta$ and then establish formula (33) by using polar coordinates near each vertex $Q_{i}$ and by solving a one-dimensional eigenvalue problem. In the course of the proof, we denote

$$
F_{i}(\bar{u})^{2}=\frac{\int_{\mathcal{B}_{Q_{i}}\left(C_{0} / 2\right)}(\nabla \bar{u})^{T} A(\nabla \bar{u}) \mathrm{d} S}{\int_{\mathcal{B}_{Q_{i}}\left(C_{0} / 2\right)} \frac{\bar{u}^{2}}{\left(x-x_{i}, y-y_{i}\right) A^{-1}\left(x-x_{i}, y-y_{i}\right)^{T}} \mathrm{~d} S},
$$


for $\bar{u} \in H_{\mathrm{D}}^{1}(\Omega), \bar{u} \neq 0$, and take the positive root. By convention, we set $F_{i}(\bar{u})=\infty$ if $\bar{u}$ is identically zero in $\mathcal{B}_{Q_{i}}\left(C_{0} / 2\right)$. Estimate (20) shows that $\inf _{\bar{u} \in H_{\mathrm{D}}^{1}(\Omega)} F_{i}(\bar{u})>0$ for each $Q_{i} \in \mathcal{V}$.

We recall the weight function $\vartheta$ is given in (15). If $0<a<\inf _{\bar{u} \in H_{\mathrm{D}}^{1}(\Omega)} F_{i}(\bar{u})$ for each $i$, then

$$
\begin{aligned}
& \int_{\mathcal{B}_{Q_{i}}\left(C_{0} / 2\right)}(\nabla \bar{u})^{T} A(\nabla \bar{u}) \mathrm{d} S-a^{2} \int_{\mathcal{B}_{Q_{i}}\left(C_{0} / 2\right)} \frac{\bar{u}^{2}}{\left(x-x_{i}, y-y_{i}\right) A^{-1}\left(x-x_{i}, y-y_{i}\right)^{T}} \mathrm{~d} S \\
\geq & \left(1-\frac{a^{2}}{\inf _{\bar{u} \in H_{\mathrm{D}}^{1}(\Omega)} F_{i}^{2}(\bar{u})}\right) \int_{\mathcal{B}_{Q_{i}}\left(C_{0} / 2\right)}(\nabla \bar{u})^{T} A(\nabla \bar{u}) \mathrm{d} S \geq C\|\nabla \bar{u}\|_{L^{2}\left(\mathcal{B}_{Q_{i}}\left(C_{0} / 2\right)\right)}^{2}
\end{aligned}
$$

for some constant $C>0$ depending on $\Omega, a$, and $A$, so that for all non-zero $\bar{u} \in H_{\mathrm{D}}^{1}(\Omega)$ it follows from (19) that

$$
B_{a}[\bar{u}, \bar{u}] \geq \beta\|\nabla \bar{u}\|_{L^{2}(\Omega)}^{2}-\gamma\|\bar{u}\|_{L^{2}(\Omega)}^{2}
$$

for some $\beta, \gamma>0$. We choose $\bar{u}=u \vartheta^{a}$, where $u$ is the weak solution of the elliptic problem (10). Then,

$$
B_{a}[\bar{u}, v]=\langle\bar{f}, v\rangle \quad \forall v \in H_{\mathrm{D}}^{1}(\Omega)
$$

with $\bar{f}=f / \vartheta^{a}$. Next, we prove that $\|\bar{u}\|_{L^{2}(\Omega)} \leq C\|\bar{f}\|_{\left(H_{\mathrm{D}}^{1}(\Omega)\right)^{\prime}}$ for some constant $C>0$. In fact, by contradiction suppose there exist sequences $\left\{\bar{f}_{k}\right\}_{k=1}^{\infty} \subset\left(H_{\mathrm{D}}^{1}(\Omega)\right)^{\prime}$ and $\left\{\bar{u}_{k}\right\}_{k=1}^{\infty} \subset H_{\mathrm{D}}^{1}(\Omega)$ such that

$$
\left\|\bar{u}_{k}\right\|_{L^{2}(\Omega)}>k\left\|\bar{f}_{k}\right\|_{\left(H_{\mathrm{D}}^{1}(\Omega)\right)^{\prime}}, \quad B_{a}\left[\bar{u}_{k}, v\right]=\left\langle\bar{f}_{k}, v\right\rangle \quad \forall v \in H_{\mathrm{D}}^{1}(\Omega) .
$$

Without loss of generality, we may assume $\left\|\bar{u}_{k}\right\|_{L^{2}(\Omega)}=1$, so that $\bar{f}_{k} \rightarrow 0$ in $\left(H_{\mathrm{D}}^{1}(\Omega)\right)^{\prime}$. By (35) and Cauchy's inequality,

$$
\begin{aligned}
\beta\left\|\nabla \bar{u}_{k}\right\|_{L^{2}(\Omega)}^{2}-\gamma\left\|\bar{u}_{k}\right\|_{L^{2}(\Omega)}^{2} & \leq B_{a}\left[\bar{u}_{k}, \bar{u}_{k}\right]=\left\langle\bar{f}_{k}, \bar{u}_{k}\right\rangle \\
& \leq C\left(\left\|\bar{u}_{k}\right\|_{H_{\mathrm{D}}^{1}(\Omega)}^{2}+\left\|\bar{f}_{k}\right\|_{\left(H_{\mathrm{D}}^{1}(\Omega)\right)^{\prime}}^{2}\right) .
\end{aligned}
$$

The sequence $\left\{\bar{u}_{k}\right\}_{k=1}^{\infty}$ is therefore bounded in $H_{\mathrm{D}}^{1}(\Omega)$ and there exists a subsequence satisfying

$$
\bar{u}_{k_{j}} \rightarrow \bar{u}_{0} \quad \text { in } H_{\mathrm{D}}^{1}(\Omega), \quad \bar{u}_{k_{j}} \rightarrow \bar{u}_{0} \quad \text { in } L^{2}(\Omega) .
$$

In particular, $\left\|\bar{u}_{0}\right\|_{L^{2}(\omega)}=1$. Since $\bar{f}_{k} \rightarrow 0$ in $\left(H_{\mathrm{D}}^{1}(\Omega)\right)^{\prime}$, we deduce

$$
B_{a}\left[\bar{u}_{0}, v\right]=0 \quad \forall v \in H_{\mathrm{D}}^{1}(\Omega),
$$


which implies $\bar{u}_{0}=0$, a contradiction. From (36) it follows that $\|\bar{u}\|_{H_{\mathrm{D}}^{1}(\Omega)} \leq$ $C\|\bar{f}\|_{\left(H_{\mathrm{D}}^{1}(\Omega)\right)^{\prime}}$, or equivalently

$$
\|u\|_{\mathcal{K}_{a+1}^{1}(\Omega)} \leq\|f\|_{\left(\mathcal{K}_{a+1}^{1}(\Omega)\right)^{\prime}}
$$

Since by Theorem 1 , we have solvability in $\mathcal{K}_{1+a}^{1}$ for $0<a<\eta$, it follows that

$$
\eta \geq \inf _{Q_{i} \in \mathcal{V}_{1}, \bar{u} \in H_{\mathrm{D}}^{1}(\Omega), \bar{u} \neq 0}\left(\frac{\int_{\mathcal{B}_{Q_{i}}\left(C_{0} / 2\right)}(\nabla \bar{u})^{T} A(\nabla \bar{u}) \mathrm{d} S}{\int_{Q_{i}\left(C_{0} / 2\right)} \frac{\bar{u}^{2}}{\left(x-x_{i}, y-y_{i}\right) A^{-1}\left(x-x_{i}, y-y_{i}\right)^{T}} \mathrm{~d} S}\right)^{1 / 2}=: \bar{F} .
$$

From the proof of Theorem 2 we have that $\delta$ is chosen to satisfy (21). Therefore, $\max \delta=\eta$ by (35) above.

Next, we are going to show that $\bar{F}$ is the optimal value for $\eta$, that is, $\eta \leq \bar{F}$. To establish this upper bound, we will explicitly compute $\bar{F}$, using the special form of the operator $L$.

First, we can reduce to the case when $L$ is a multiple of the Laplacian in each subdomain $\Omega_{k}, k=1, \ldots, K$ by essentially diagonalizing the matrix $A$. We fix a vertex $Q_{i} \in \mathcal{V}$. Since formula (33) is local near each vertex, we are only interested in the part of the interface that joins $Q_{i}$ (see Fig. 2, top vertex). As in the proof of Theorem 2 , we denote the subdomains adjacent to the vertex $Q_{i}$ by $\Omega_{i l}, l=1, \ldots, K(i)$. Let $\omega_{i}$ denote the aperture angle of the corner with vertex $Q_{i}$ in $\Omega$, and let $(r, \phi)$ denote polar coordinates centered at $Q_{i}$ with main axis along one of the Dirichlet sides of the corner. Because we assume that $\mathcal{V}_{2}=\emptyset$, there is always at least one side of the corner with vertex $Q_{i}$ with Dirichlet boundary conditions. Then sufficiently close to the vertex $Q_{i}$, each subdomain $\Omega_{i l}$ is a sector centered at $Q_{i}$ with angle $\omega_{i l+1}-\omega_{i l}$, $l=0, \ldots, K(i)$, where $\omega_{i, 0}=0$ and $\omega_{i, K(i)}=\omega_{i}$. Without loss of generality, we can label the vectors in such a way that $0=\omega_{i 0}<\omega_{i 1}<\cdots<\omega_{i K(i)}=\omega_{i}$. We recall the definition of $\mathcal{B}_{Q_{i}}\left(C_{0} / 2\right)$ :

$$
\begin{aligned}
\mathcal{B}_{Q_{i}}\left(C_{0} / 2\right)= & \left\{(x, y) \mid \vartheta_{i}(x, y)=C_{i l}\left\{\left(x-x_{i}, y-y_{i}\right) A^{-1}\left(x-x_{i}, y-y_{i}\right)^{T}\right\}^{1 / 2}\right. \\
& \left.\leq C_{0} / 2\right\}
\end{aligned}
$$

We next diagonalize the matrix $A=A_{i l}$ in each $\Omega_{i l}$ (recall $A$ is symmetric and definite positive) via an orthogonal matrix $R_{i l}$ and then apply a dilation matrix $\Lambda_{i l}$ to rescale the eigenvalues to be all equal to a positive constant $d_{i l}$. We set $\Phi_{i l}=\Lambda_{i l} R_{i l}$. We introduce a new coordinate system $\left(r^{\prime}, \phi^{\prime}\right)$ obtained by applying $\Phi_{i l}$ in each sector $\Omega_{i l} \cap \mathcal{B}_{Q_{i}}\left(C_{0} / 2\right):$

$\left(r^{\prime} \cos \left(\phi^{\prime}\right), r^{\prime} \sin \left(\phi^{\prime}\right)\right)=\Phi_{i l}(r \cos (\phi), r \sin (\phi)), \quad \phi \in\left(\omega_{i l}, \omega_{i, l+1}\right), \quad 0<r<C_{0} / 2$. 
We choose the constants $d_{i l}$ so that this transformation is continuous across the interface between different sectors and fixes the Dirichlet side corresponding to $\phi=0$. That is, we take the matrix $\Phi$ satisfying:

(i) $\operatorname{Det}\left(\Phi_{i l}\right)>0$;

(ii) $\Phi_{i 1}(r, 0)=(r, 0)$;

(iii) $\Phi_{i l}\left(r \cos \left(\omega_{i l}\right), r \sin \left(\omega_{i l}\right)\right)=P_{i} l+1\left(r \cos \left(\omega_{i l}\right), r \sin \left(\omega_{i l}\right)\right), l=1,2, \ldots, L-1$;

(iv) $\Phi_{i l} A_{i l} \Phi_{i l}^{T}=d_{i l} I$ for some constant $d_{i l}>0$.

These requirements uniquely determine $\Phi$. Under the transformation $\Phi$, each sector $\Omega_{i l} \cap \mathcal{B}_{Q_{i}}\left(C_{0} / 2\right)$ is mapped onto another sector centered at $Q_{i}$ :

$$
\mathcal{B}_{Q_{i}}^{\prime}\left(r_{, l}^{\prime} / 2\right)=\left\{\left(r^{\prime}, \phi^{\prime}\right) \mid \frac{C_{i l}}{\sqrt{d_{i l}}} r^{\prime} \leq C_{0} / 2, \omega_{i l-1}^{\prime} \leq \phi^{\prime} \leq \omega_{i l}^{\prime}\right\},
$$

with $0=\omega_{0}^{\prime}<\omega_{i 1}^{\prime}<\omega_{i 2}^{\prime}<\cdots<\omega_{i L}^{\prime}=\omega_{i}^{\prime}<2 \pi$, and $r_{i, l}^{\prime}=\frac{C_{0} \sqrt{d_{i l}}}{C_{i l}}$ for each $l$. Because of Condition 5 on $\Phi, r_{i, l}^{\prime}=r_{i, l+1}$ and we denote this common value simply as $r_{0}^{\prime}$, suppressing the dependence of $i$.

Let now $\bar{u} \in H_{\mathrm{D}}^{1}(\Omega)$. With slight abuse of notation, we write $\bar{u}(r, \phi)$ for $\bar{u}\left(r \cos (\phi), r \sin (\phi)\right.$ in $\mathcal{B}_{Q_{i}}\left(C_{0} / 2\right)$. We let $\hat{u}\left(r^{\prime}, \phi^{\prime}\right)$ be the image of $\bar{u}(r, \phi)$. Then $\hat{u}$ is well defined on the sector $\mathcal{B}_{Q_{i}}^{\prime}\left(r_{0}^{\prime}\right)$ and $(\nabla \hat{u})=\left(\Phi^{-1}\right)^{T}(\nabla \bar{u})$. A simple change of variables show that $F_{i}^{2}(\bar{u})$ is transformed into:

$$
\hat{F}_{i}^{2}(\hat{u})=\frac{\sum_{l=1}^{K(i)}\left(\operatorname{Det}\left(A_{i l}\right)\right)^{1 / 2}\left(\left\|\hat{u}_{r^{\prime}}\right\|_{L^{2}\left(\mathcal{B}_{Q_{i}}^{\prime}\left(r_{0}^{\prime} / 2\right) \cap \Omega_{i l}\right)}^{2}+\left\|\hat{u}_{r^{\prime}} / r^{\prime}\right\|_{L^{2}\left(\mathcal{B}_{Q_{i}}^{\prime}\left(r_{0}^{\prime} / 2\right) \cap \Omega_{i l}\right)}^{2}\right)}{\sum_{l=1}^{K(i)}\left(\operatorname{Det}\left(A_{i l}\right)\right)^{1 / 2}\left\|\hat{u} / r^{\prime}\right\|_{L^{2}\left(\mathcal{B}_{Q_{i}}^{\prime}\left(r_{0}^{\prime} / 2\right) \cap \Omega_{i l}\right)}^{2}} .
$$

It is also straightforward to show that, if $\bar{u}$ satisfies (16), then locally near the vertex $Q_{i}, \hat{u}$ is harmonic in each sector $\omega_{i, l}^{\prime}<\phi^{\prime}<\omega_{i, l+1}^{\prime}, l=0, \ldots, K(i)-1$, satisfies homogeneous Dirichlet or homogeneous Neumann conditions on the sides $\phi^{\prime}=\omega_{0}^{\prime}=$ 0 and $\phi^{\prime}=\omega_{i}^{\prime}$, depending on whether the vertex $Q_{i}$ was a Dirichlet-Dirichlet or a Dirichlet-Neumann vertex, and satisfies standard transmission conditions (continuity of $\hat{u}$ and its conormal derivative) at $\phi^{\prime}=\omega_{i l}^{\prime}, l=1, \ldots, K(i)-1$.

Our task is therefore reduced to computing $F_{i}(\bar{u})$ for each $Q_{i} \in \mathcal{V}$, in the case that the operator $L$ is a constant multiple of the Laplacian, $L=-C_{k} \triangle, C_{k}>0$, in each subdomain $\Omega_{k}, k=1, \ldots, K$. Passing to polar coordinates $(r, \phi)$ again near the vertex $Q_{i}$ gives:

$$
\begin{aligned}
F_{i}^{2}(\bar{u}) & =\frac{\sum_{l=1}^{K(i)} C_{i l}\left(\left\|\bar{u}_{r}\right\|_{L^{2}\left(\mathcal{B}_{Q_{i}}\left(C_{0} / 2\right) \cap \Omega_{i l}\right)}^{2}+\left\|\bar{u}_{\phi} / r\right\|_{L^{2}\left(\mathcal{B}_{Q_{i}}\left(C_{0} / 2\right) \cap \Omega_{i l}\right)}^{2}\right)}{\sum_{l=1}^{K(i)} C_{i l}\|\bar{u} / r\|_{L^{2}\left(\mathcal{B}_{Q_{i}}\left(C_{0} / 2\right) \cap \Omega_{i l}\right)}^{2}} \\
\geq & \frac{\sum_{l=1}^{K(i)} C_{i l}\left\|\bar{u}_{\phi}\right\|_{L^{2}\left(\mathcal{B}_{Q_{i}}\left(C_{0} / 2\right) \cap \Omega_{i l}\right)}^{2}}{\sum_{l=1}^{K(i)} C_{i l}\|\bar{u}\|_{L^{2}\left(\mathcal{B}_{Q_{i}}\left(C_{0} / 2\right) \cap \Omega_{i l}\right)}^{2}} .
\end{aligned}
$$


To minimize the right-hand side of (40), we consider a minimization problem for functions of the angle $0<\phi<\omega_{i}$. Specifically, we characterize

$$
\min _{\tilde{u} \in H^{1}[0, \omega], \tilde{u}(0)=0} \frac{\sum_{l=1}^{K(i)} \int_{\omega_{i l-1}}^{\omega_{i l}} C_{i l} \tilde{u}_{\phi}^{2} d \phi}{\sum_{l=1}^{K(i)} \int_{\omega_{i l-1}}^{\omega_{i l}} C_{i l} \tilde{u}^{2} d \phi}
$$

when $Q_{i}$ is a Neumann-Dirichlet vertex, or

$$
\min _{\tilde{u} \in H^{1}[0, \omega], \tilde{u}(0)=\tilde{u}(\omega)=0} \frac{\sum_{l=1}^{K(i)} \int_{\omega_{i l-1}}^{\omega_{i l}} C_{i l} \tilde{u}_{\phi}^{2} d \phi}{\sum_{l=1}^{K(i)} \int_{\omega_{i l-1}}^{\omega_{i l}} C_{i l} \tilde{u}^{2} d \phi}
$$

when $Q_{i}$ is a Dirichlet-Dirichlet vertex. The 1D Poincaré's inequality guarantees that the infimum is strictly positive in both cases. We denote it by $\lambda_{i 0}^{2}$. We will prove that the minimum is achieved.

We assume first that $Q_{i}$ is a Neumann-Dirichlet vertex. Suppose that $\tilde{u} \in H^{1}[0, \omega]$ is the minimizer of (41), then it is a stationary point of the functional in (41), which gives:

$$
\frac{\sum_{l=1}^{K(i)} \int_{\omega_{i l-1}}^{\omega_{i l}} C_{i l} \tilde{u}_{\phi}^{2} d \phi}{\sum_{l=1}^{K(i)} \int_{\omega_{i l-1}}^{\omega_{i l}} C_{i l} \tilde{u}^{2} d \phi}=\frac{\sum_{l=1}^{K(i)} \int_{\omega_{i l-1}}^{\omega_{i l}} C_{i l} \tilde{u}_{\phi} v_{\phi} d \phi}{\sum_{l=1}^{K(i)} \int_{\omega_{i l-1}}^{\omega_{i l}} C_{i l} \tilde{u} v d \phi}=\lambda_{i 0}^{2}
$$

for all $v \in H^{1}[0, \omega]$ with $v(0)=0$. Integrating by parts,

$$
\begin{aligned}
& \sum_{l=1}^{K(i)} \int_{\omega_{i l-1}}^{\omega_{i l}} C_{i l} \tilde{u}_{\phi} v_{\phi} d \phi=-\sum_{l=1}^{K(i)} \int_{\omega_{i l-1}}^{\omega_{i l}} C_{i l} \tilde{u}_{\phi \phi} v d \phi \\
& \quad+\sum_{l=1}^{L-1}\left(C_{i l+1} \tilde{u}_{\phi}^{+}\left(\omega_{l}\right)-C_{i l} \tilde{u}_{\phi}^{-}\left(\omega_{l}\right)\right) v\left(\omega_{l}\right)+C_{i L} \tilde{u}_{\phi}\left(\omega_{i}\right) v\left(\omega_{i}\right) .
\end{aligned}
$$

As $v$ is arbitrary, $\tilde{u}$ is the unique weak solution to the following eigenvalue problem

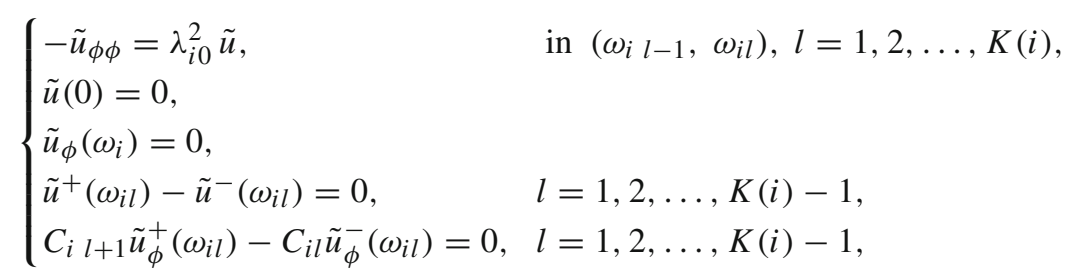

with $\lambda_{i 0}^{2}$ the smallest eigenvalue. We can solve explicitly this $1 \mathrm{D}$ eigenvalue problem to show the existence of a unique minimizer. 
Similarly, for a Dirichlet-Dirichlet vertex $Q_{i}$, the minimizer $\tilde{u}$ satisfies the following eigenvalue problem for the minimal eigenvalue:

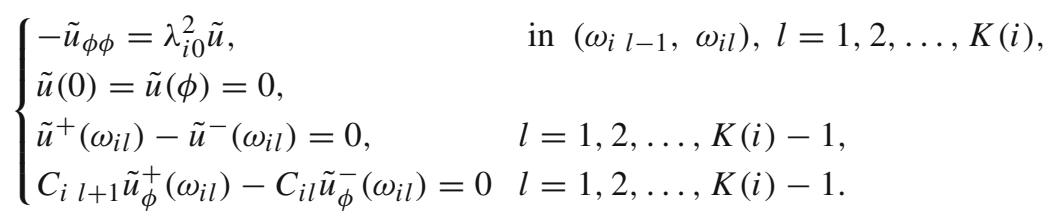

Again, the existence of a unique minimizer can be established by ODE methods.

Now, a direct calculation shows that $\lambda_{i 0}^{2}$ is a lower bound for $F_{i}^{2}(\bar{u})$

$$
F_{i}^{2}(\bar{u}) \geq \frac{\sum_{l=1}^{K(i)} C_{i l}\left\|\bar{u}_{\phi}\right\|_{\mathcal{B}_{Q_{i}}\left(C_{0} / 2\right) \cap \Omega_{i l}}^{2}}{\sum_{l=1}^{K(i)} C_{i l}\|\bar{u}\|_{\mathcal{B}_{Q_{i}}\left(C_{0} / 2\right) \cap \Omega_{i l}}^{2}} \geq \lambda_{i 0}^{2},
$$

We prove next that it is actually the infimum over all restrictions to the sector $\mathcal{B}_{Q_{i}}\left(C_{0} / 2\right)$ of functions $\bar{u} \in H_{\mathrm{D}}^{1}(\Omega)$. To this end, for each $\epsilon>0$ we let

$$
\bar{u}(r, \phi)=\chi(r) r^{\epsilon} \tilde{u}(\phi) \in H_{\mathrm{D}}^{1}(\Omega),
$$

where $\chi(r)$ is a smooth cut-off function that it equals 1 on $\left[0, C_{0} / 2\right]$, and equals 0 on $\left[C_{0}, \infty\right)$. We have then:

$$
\begin{aligned}
\lambda_{i 0}^{2} & \leq \inf _{\bar{u} \in H_{\mathrm{D}}^{1}(\Omega)} F_{i}^{2}(\bar{u}) \\
& =\inf _{\bar{u} \in H_{\mathrm{D}}^{1}(\Omega)} \frac{\sum_{l=1}^{K(i)} C_{i l}\left(\left\|\bar{u}_{r}\right\|_{L^{2}\left(\mathcal{B}_{Q_{i}}\left(C_{0} / 2\right) \cap \Omega_{i l}\right)}^{2}+\left\|\bar{u}_{\phi} / r\right\|_{L^{2}\left(\mathcal{B}_{Q_{i}}\left(C_{0} / 2\right) \cap \Omega_{i l}\right)}^{2}\right)}{\sum_{l=1}^{L} C_{i l}\|\bar{u} / r\|_{L^{2}\left(\mathcal{B}_{Q_{i}}\left(C_{0} / 2\right) \cap \Omega_{i l}\right)}^{2}} \\
& \leq \lim _{\epsilon \rightarrow 0} \frac{\sum_{l=1}^{L} C_{i l}\left(\left\|\epsilon r^{\epsilon-1} \tilde{u}(\phi)\right\|_{L^{2}\left(\mathcal{B}_{Q_{i}}\left(C_{0} / 2\right) \cap \Omega_{i l}\right)}^{2}+\left\|r^{\epsilon-1} \tilde{u}_{\phi}(\phi)\right\|_{L^{2}\left(\mathcal{B}_{Q_{i}}\left(C_{0} / 2\right) \cap \Omega_{i l}\right)}^{2}\right)}{\sum_{l=1}^{L} C_{i l}\left\|r^{\epsilon-1} \tilde{u}(\phi)\right\|_{L^{2}\left(\mathcal{B}_{Q_{i}}\left(C_{0} / 2\right) \cap \Omega_{i l}\right)}^{2}} \\
& \leq \lim _{\epsilon \rightarrow 0} \frac{\sum_{l=1}^{L} C_{i l}\left\|r^{\epsilon-1} \tilde{u}_{\phi}(\phi)\right\|_{L^{2}\left(\mathcal{B}_{Q_{i}}\left(C_{0} / 2\right) \cap \Omega_{i l}\right)}^{2}}{\sum_{l=1}^{L} C_{i l}\left\|r^{\epsilon-1} \tilde{u}(\phi)\right\|_{L^{2}\left(\mathcal{B}_{Q_{i}}\left(C_{0} / 2\right) \cap \Omega_{i l}\right)}^{2}}=\frac{\sum_{l=1}^{L} \int_{\omega_{i l} l-1}^{\omega_{i l}} C_{i l} \tilde{u}_{\phi}^{2} \mathrm{~d} \phi}{\sum_{l=1}^{L} \int_{\omega_{i l-1}}^{\omega_{i l}} C_{i l} \tilde{u}^{2} \mathrm{~d} \phi}=\lambda_{i 0}^{2} .
\end{aligned}
$$

Therefore

$$
\inf _{\bar{u} \in H_{\mathrm{D}}^{1}(\Omega), \bar{u} \neq 0} F_{i}(\bar{u})=\min _{Q_{i} \in \mathcal{V}} \lambda_{i 0},
$$

It remains to show that $\eta \leq \lambda_{i 0}$ for all $Q_{i} \in \mathcal{V}$. We construct a weak solution of Problem 10 for a suitable $f$, for which the regularity result in Theorem 1 does not hold if $\eta>\lambda_{i 0}$ for some $Q_{i} \in \mathcal{V}$. In fact, we pick $f=0$ in (10) and choose $a=\lambda_{i 0}$. We let

$$
\bar{u}(r, \phi)=\chi(r) r^{\lambda_{i 0}} \tilde{u}_{\phi}(\phi)
$$


where $\chi_{i}(r)$ is a smooth cut-off function that equals 1 on the interval $\left[0, C_{0} / 2\right]$ and vanishes on $\left[C_{0}, \infty\right)$. Then $\bar{u}$ is supported on the sector $\mathcal{B}_{Q_{i}}\left(C_{0}\right)$, and

(1) $\bar{u}$ satisfies the boundary conditions and the transmission conditions on the interface.

(2) $\Delta \bar{u}=0$ on the sectors $\mathcal{B}_{Q_{i}}\left(C_{0} / 2\right) \cap \Omega_{i l}$, which implies $\Delta \bar{u}=0$ in $\mathcal{K}_{\lambda_{i 0}}^{0}(\Omega)$.

(3) $\bar{u} \notin \mathcal{K}_{\lambda_{i 0}+1}^{1}(\Omega)$, since $1 / r \notin L^{2}(\Omega)$.

This example can be modified to show that, for the parabolic problem (3), necessarily $\delta \leq \lambda_{i 0}$ for all $Q_{i} \in \mathcal{V}$. We omit the details.

Example 1 Assume $\Omega$ has interior aperture angle $\pi / 4$ at one of its vertices $Q$, and the domain is split into two sub-polygons $\Omega_{1}$ and $\Omega_{2}$. Also, we assume the coefficient matrix $A$ takes values

$$
A_{1}=\left(\begin{array}{cc}
1 & -1 \\
-1 & 2
\end{array}\right), \quad A_{2}=\left(\begin{array}{ll}
100 & 100 \\
100 & 200
\end{array}\right)
$$

in $\Omega_{1}$ and $\Omega_{2}$ respectively, Then, the transformation matrix $\Phi$ takes the form

$$
\Phi_{1}=\left(\begin{array}{ll}
1 & 1 / 2 \\
0 & 1 / 2
\end{array}\right), \quad \Phi_{2}=\left(\begin{array}{cc}
2 & -1 / 2 \\
-1 & 3 / 2
\end{array}\right)
$$

with corresponding values $d_{1}=1 / 2, d_{2}=250$. Moreover,

$$
\Phi_{1}\left(\begin{array}{l}
1 \\
0
\end{array}\right)=\left(\begin{array}{l}
1 \\
0
\end{array}\right), \quad \Phi_{1}\left(\begin{array}{l}
1 \\
1
\end{array}\right)=\Phi_{2}\left(\begin{array}{l}
1 \\
1
\end{array}\right)=\left(\begin{array}{c}
3 / 2 \\
1 / 2
\end{array}\right), \quad \Phi_{2}\left(\begin{array}{c}
-1 \\
1
\end{array}\right)=\left(\begin{array}{c}
-5 / 2 \\
5 / 2
\end{array}\right)
$$

so that $\omega_{1}^{\prime}=\arctan (1 / 3)$ and $\omega_{2}^{\prime}=3 \pi / 4$. The value of $\lambda_{0}$ is determined by solving the following eigenvalue problem

$$
\begin{cases}-\tilde{u}_{\phi^{\prime} \phi^{\prime}}=\lambda_{0}^{2} \tilde{u}, & \text { in }\left(0, \omega_{1}^{\prime}\right) \cup\left(\omega_{1}^{\prime}, 3 \pi / 4\right), \\ \tilde{u}(0)=0, \quad \tilde{u}_{\phi^{\prime}}(3 \pi / 4)=0, & \\ \tilde{u}^{+}\left(\omega_{1}^{\prime}\right)-\tilde{u}^{-}\left(\omega_{i 1}^{\prime}\right)=0, \quad \tilde{u}_{\phi^{\prime}}^{+}\left(\omega_{i 1}^{\prime}\right)-100 \tilde{u}_{\phi^{\prime}}^{-}\left(\omega_{i 1}^{\prime}\right)=0 . & \end{cases}
$$

which gives $\lambda_{0} \approx 0.1332$.

Remark 5 One common approach to constructing solutions of parabolic problems, even for semi-linear and some quasi-linear problem is by semi-group methods (see e.g. [47]). It is a natural question whether the operator $L$ generates a $C^{0}$ semigroup on the weighted space $\mathcal{K}_{a}^{0}(\Omega)$. There are indications that this is not the case (we refer to [53] for further discussion on this point). We reserve to study this problem further in future work.

\section{Applications to the FEM for Parabolic Problems}

In two final sections, we discuss applications of the regularity results in weighted spaces for the IBVTP (3) to the implementation of the FEM. We use the FEM only 
Fig. 3 Uniform triangular mesh

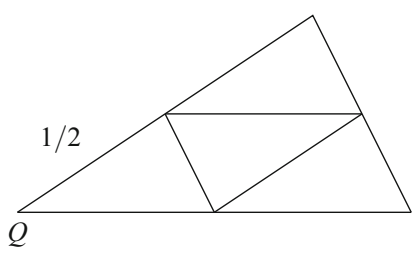

Fig. 4 Mesh refinement with grading

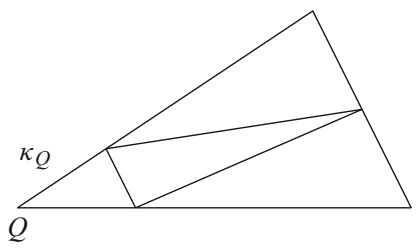

for the space discretization, while we implement the backward Euler method in time. We do not assume $\mathcal{V}_{2}=\emptyset$. In particular, we present numerical tests where NeumannNeumann vertices and singular interfaces are present. The numerical scheme is not optimized-for instance, we do not make our method adaptive, though adaptivity is possible - as our examples have only pedagogical purpose.

We utilize a known open-source package, FEM2d_HEAT by J. Alberty, C. Carstensen and S. A. Funken [4], implemented in MATLAB ${ }^{\odot}$, but adapted to the operators and the geometric set-up considered in this work. In this package, the mass matrix is inverted via an $L U$ decomposition. We choose to employ a backward first-order scheme in time for numerical efficiency and stability.

Our point of departure is a particular implementation of the FEM on polygonal domains for mixed-boundary-value-transmission elliptic problems that uses graded meshes and yields quasi-optimal rates of convergence [32]. Here we extend this scheme to the parabolic case and prove quasi-optimal rates of convergence for a mixed fully discrete scheme. There is an extensive literature on implementing the FEM on polygonal domains with focus on rates of convergence. We refer to the discussion in the Introduction. The graded mesh is obtained from a sequence of triangulations $\left\{\mathcal{T}_{n}\right\}_{n \geq 0}$ of $\Omega$ with the property that $\mathcal{T}_{n}$ consists of uniform triangles away from the vertices, but the triangles are graded near the vertices. The grading depends on the order $m$ of the polynomials used for the FEM approximation and on the singularity constant $\eta$ in Theorem 1 . The grading is obtained as follows. Let $\mathcal{T}_{0}$ be a given uniform triangulation of $\Omega$ such that each each vertex in $\mathcal{V}$ is also a vertex in $\mathcal{T}_{0}$, each triangle in $\mathcal{T}_{0}$ contains only one vertex in $\mathcal{V}$ and the sides of the interface $\Gamma$ are also sides of triangles in $\mathcal{T}_{0}$. We also choose the size of each initial triangle small enough. Then $\mathcal{T}_{n}$ is constructed from $\mathcal{T}_{n-1}$ inductively. Let $T$ be a triangle in $\mathcal{T}_{n-1}$ and let $A$ be a vertex of $T$. We divide the side $A B$ (and similarly for the other side of the triangle with vertex at $A$ ) according to the following rule. If $A=Q \in \mathcal{V}$ then we divide $A B$ into $A C$ and $C B$ such that $|A C|=\kappa_{Q}|A B|$ for a certain constant $\kappa_{Q}$, otherwise we divide the side $A B$ into two equal parts (see Figs. 3,4 ). The positive constant $\kappa_{Q}$ cannot exceed $2^{-m} \eta_{Q}$, where $\eta_{Q}$ is the singularity constant near the vertex $Q$, which can be computed by using Theorem 5. In fact, $\eta_{Q}=\lambda_{i 0}$ in the notation of that proof of Theorem 5, where 
$Q=Q_{i}$. So, the grading is adapted to each vertex and there might not be any special grading at some vertex, as exemplified in our numerical tests. The refinement procedure and the choice of initial mesh guarantees that at each step in the refinement each vertex in $\mathcal{V}$ belongs to a triangle in the mesh and the interface is aligned with sides of elements of the mesh. So, no triangles in $\mathcal{T}_{n}$ can cross the interface. This refinement also preserves the quality of the mesh.

We let $S_{n}:=S\left(\mathcal{T}_{n}, m\right) \subset H_{\mathrm{D}}^{1}(\Omega)$ denote the finite element space of continuous functions on $\Omega$ that restrict to a polynomial of degree $m \geq 1$ on each triangle of $\mathcal{T}_{n}$. We recall the treatment of the elliptic problem (10) from [32]. We let $u_{n} \in S_{n}$ be the finite element approximation of the weak solution $u$, which is the exact solution of the variational problem

$$
B\left[u_{n}, v_{n}\right]:=\int_{\Omega} A_{i j} \partial_{i} u_{n} \partial_{j} v_{n} \mathrm{~d} x=\left(f, v_{n}\right) \quad \forall v_{n} \in S\left(\mathcal{T}_{n}, m\right) .
$$

Then the following quasi-optimal rates of convergence hold for the FEM approximation:

$$
\left\|u-u_{n}\right\|_{H^{1}(\Omega)} \leq C^{\prime}\left\|u-u_{n}\right\|_{\mathcal{K}_{1}^{1}(\Omega)} \leq C 2^{-n m}\|f\|_{\hat{\mathcal{K}}_{a-1}^{m-1}(\Omega)},
$$

and

$$
\left\|u-u_{n}\right\|_{L^{2}(\Omega)} \leq C 2^{-n(m+1)}\|f\|_{\hat{\mathcal{K}}_{a-1}^{m-1}(\Omega)},
$$

for some constant $C>0$. The rates in (49)-(50) are optimal in the sense that they are the rates valid on smooth domains, where the right hand side can be replaced by $\|u\|_{H^{m+1}(\Omega)}$ in that case. The quasi-optimality stems from the fact that we do not optimize constants.

We next tackle the numerical approximation of the parabolic problem (3). We are going to implement a mixed fully-discrete scheme, consisting in a FEM approximation in space and a finite-difference approximation in time, using backward Euler's method. We confine ourselves to a first-order method, though unconditionally stable, to reduce the computational cost. The numerical tests we perform have the sole purpose of validating the theoretical result.

Let $u$ denote the weak solution of the IVBPT (3) on the time interval $[0, T]$. After $N$ refinement of the mesh, we shall denote the maximum diameter of triangles in $\mathcal{T}_{n}$ by $h$, the space step size. Similarly, we shall denote by $k$ the size of the time step after $M$ steps, i.e., $k=T / M$. The numerical approximation $u_{n}^{j} \in S_{n}, n=0, \ldots, N$, $j=0, \ldots, M$, of $u$ is the solution of the following coupled system:

$$
\begin{aligned}
& \left(\frac{u_{n}^{j+1}-u_{n}^{j}}{k}, v_{n}\right)+B\left[u_{n}^{j+1}, v_{n}\right]=\left(f^{j+1}, v_{n}\right) \quad \forall v_{n} \in S_{n}, \\
& \left(u_{n}^{0}, v_{n}\right)=\left(g, v_{n}\right) \quad \forall v_{n} \in S_{n},
\end{aligned}
$$

where $g \in L^{2}(\Omega)$ is the initial condition for the exact problem (3) and $f^{j}(\cdot)=$ $f(\cdot, j k)$ with $f \in C\left([0, T], H_{\mathrm{D}}^{1}(\Omega)^{\prime}\right)$. 
Our main results in this section are an extension of the quasi-optimal rates of convergence to the parabolic setting, using the mixed, fully discrete scheme. One could also study convergence for a semi-discrete scheme, which is FEM in space and continuous in time. We consider only the case of piecewise-linear or quadratic polynomials, as higher-order polynomials require higher regularity for the solution in weighted spaces, which is turn requires higher compatibility conditions on the data.

Theorem 6 Let $u$ be the weak solution of the IBPTP (3) on [0,T]. Assume $0<a<$ $\min _{Q \in \mathcal{V}}\left\{\eta_{Q}\right\}$ and

$$
\begin{gathered}
f_{t t} \in L^{2}\left(0, T ;\left(H_{\mathrm{D}}^{1}(\Omega)\right)^{\prime}\right), f_{t} \in C\left(0, T ; L^{2}(\Omega)\right), f \in C\left(0, T ; \hat{\mathcal{K}}_{a}^{2}(\Omega)\right) ; \\
g \in V_{a+1}^{2}(\Omega), g_{1}=f(0)-P g \in V_{a+1}^{2}(\Omega), g_{2}=f_{t}(0)-P g_{1} \in L^{2}(\Omega) ;
\end{gathered}
$$

Let $u_{n}^{j}, j=0, \ldots, M, n=0, \ldots, N$ the solution of the system (51)-(52) with $m=1$. Then, the following error estimates hold:

$$
\begin{aligned}
& \max _{0 \leq j \leq M}\left\|u(j k)-u_{n}^{j}\right\|_{L^{2}(\Omega)}=O\left(h^{2}+k\right), \\
& \max _{0 \leq j \leq M}\left\|u(j k)-u_{n}^{j}\right\|_{H_{\mathrm{D}}^{1}(\Omega)}=O\left(h+\frac{h^{2}+k}{\sqrt{k}}\right) .
\end{aligned}
$$

where $h=h_{n}$ is the size of the elements of the triangulation $\mathcal{T}_{n}$ and $k=k_{j}$ is the size of the time step.

Proof From Theorem 4 we can decompose $u$ as $u=u_{\text {reg }}+w_{\mathrm{s}}$, where

$$
u_{\text {reg }} \in W^{2, \infty}\left(0, T ; L^{2}(\Omega)\right) \cap W^{1, \infty}\left(0, T ; \hat{\mathcal{K}}_{a+1}^{2}(\Omega)\right) \cap L^{\infty}\left(0, T ; \hat{\mathcal{K}}_{a+1}^{4}(\Omega)\right) ;
$$

and $w_{\mathrm{s}} \in W_{\mathrm{s}}$. The rest of the proof follows a standard approach for FEM (see e.g. [7]). Since $u$ is the exact solution of (3), and $f-u_{t} \in W^{1, \infty}\left(0, T ; L^{2}(\Omega)\right)$, there exists a unique solution $w_{n}(t) \in S_{n}, t \in[0, T]$ of the problem

$$
B\left[w_{n}, v_{n}\right]=\left(f-u_{t}, v_{n}\right), \quad \forall v_{n} \in S_{n}, \quad 0<t<T,
$$

such that

$$
B\left[\partial_{t} w_{n}, v_{n}\right]=\left(f_{t}-u_{t t}, v_{n}\right), \quad \forall v_{n} \in S_{n}, 0<t<T .
$$

Since $u$ solves (3) so that $B[u, v]=\left(f-u_{t}, v\right)$ for all $v \in H_{\mathrm{D}}^{1}(\Omega)$ and $0<t<T$, we also have:

$$
B\left[w_{n}, v_{n}\right]=B\left[u, v_{n}\right], \quad \forall v_{n} \in S_{n}, 0<t<T
$$

Differentiating the above identity in time, we conclude that $\partial_{t} w_{n} \in S_{n}$ is the FEM approximation of $P^{-1}\left(f_{t}-u_{t t}\right)$, where we recall $P$ is the forward operator for the elliptic problem (10). From estimates (49), (50) with $m=1$, we deduce that 


$$
\begin{gathered}
\left\|u_{t}-\partial_{t} w_{n}\right\|_{L^{2}(\Omega)} \leq C h^{2}\left\|f_{t}-u_{t t}\right\|_{\mathcal{K}_{a-1}^{0}(\Omega)}, \\
\left\|u_{t}-\partial_{t} w_{n}\right\|_{H_{\mathrm{D}}^{1}(\Omega)} \leq C h\left\|f_{t}-u_{t t}\right\|_{\mathcal{K}_{a-1}^{0}(\Omega)}
\end{gathered}
$$

for some constant $C>0$ and $0<t<T$.

From Taylor's formula, given the regularity of $u$, it follows that

$$
\begin{aligned}
\left\|\frac{u((j+1) k)-u(j k)}{k}-u_{t}((j+1) k)\right\|_{L^{2}(\Omega)} & =\frac{k}{2}\left\|u_{t t}(j k+\epsilon)\right\|_{L^{2}(\Omega)} \\
& \leq \frac{k}{2}\left\|u_{t t}\right\|_{L^{\infty}\left(0, T ; L^{2}(\Omega)\right)}
\end{aligned}
$$

while (56a) gives

$$
\begin{aligned}
\| & {\left[w_{n}((j+1) k)-u((j+1) k]-\left[w_{n}(j k)-u(j k)\right]\right.} \\
& =\left\|\frac{1}{k} \int_{j k}^{(j+1) k}\left[\partial_{t} w_{n}(s)-u_{t}(s)\right] \mathrm{d} s\right\|_{L^{2}(\Omega)} \\
& \leq\left\|\partial_{t} w_{n}-u_{t}\right\|_{\left.L^{\infty}(\Omega) T ; L^{2}(\Omega)\right)} \leq C h^{2}\left\|f_{t}-u_{t t}\right\|_{L^{\infty}\left(0, T ; \mathcal{K}_{a-1}^{0}(\Omega)\right)} \\
& \leq C h^{2}\left\|f_{t}-u_{t t}\right\|_{L^{\infty}\left(0, T ; L^{2}(\Omega)\right)} .
\end{aligned}
$$

Now, (55) implies that for $j=0, \ldots, M$,

$$
\begin{aligned}
& \left(\frac{w_{n}((j+1) k)-w_{n}(j k)}{k}, v_{n}\right)+B\left[w_{n}((j+1) k), v_{n}\right] \\
& =B\left[u((j+1) k), v_{n}\right]+\left(\frac{u((j+1) k)-u(j k)}{k}, v_{n}\right) \\
& \quad+\left(\frac{\left[w_{n}((j+1) k)-u((j+1) k]-\left[w_{n}(j k)-u(j k)\right]\right.}{k}, v_{n}\right) .
\end{aligned}
$$

We rewrite this identity as:

$$
\begin{aligned}
& \left(\frac{w_{n}((j+1) k)-w_{n}(j k)}{k}, v_{n}\right)+B\left[w_{n}((j+1) k), v_{n}\right]=B\left[u((j+1) k), v_{n}\right]+ \\
& \left(u_{t}((j+1) k), v_{n}\right)+\left(z_{n}^{j+1}, v_{n}\right)=\left(f((j+1) k), v_{n}\right)+\left(z_{n}^{j+1}, v_{n}\right)= \\
& \left(f^{j+1}, v_{n}\right)+\left(z_{n}^{j+1}, v_{n}\right) \quad \forall v_{n} \in S_{n}, j=0,1, \ldots
\end{aligned}
$$

where, by exploiting estimates (57)-(58) above in this identity,

$$
\left\|z_{n}^{j}\right\|_{L^{2}(\Omega)} \leq C h^{2}\left\|f_{t}\right\|_{L^{\infty}\left(0, T ; L^{2}(\Omega)\right)}+\left(C h^{2}+k\right)\left\|u_{t t}\right\|_{L^{\infty}\left(0, T ; L^{2}(\Omega)\right)} \quad j=0,1, \ldots
$$


Next we compare (51) with (60), and let $y_{n}^{j}=u_{n}^{j}-w_{n}(j k), j=0,1, \ldots, M$, so that

$$
\left(\frac{y_{n}^{j+1}-y_{n}^{j}}{k}, v_{n}\right)+B\left[y_{n}^{j+1}, v_{n}\right]=\left(z_{n}^{j+1}, v_{n}\right) \quad \forall v_{n} \in S_{n}, j=0,1, \ldots
$$

For each $j=0, \ldots, M$ we choose $v_{n}=y_{n}^{j+1} \in S_{n}$ in the identity above, obtaining

$$
\left(y_{n}^{j+1}, y_{n}^{j+1}\right)+k B\left[y_{n}^{j+1}, y_{n}^{j+1}\right]=\left(y_{n}^{j}+k z_{n}^{j+1}, y_{n}^{j+1}\right)
$$

Since the bilinear form $B[$,$] is positive definite, we deduce that$

$$
\max _{0 \leq j \leq M}\left\|y_{n}^{j}\right\|_{L^{2}(\Omega)} \leq\left\|y_{n}^{0}\right\|_{L^{2}(\Omega)}+k \sum_{s=1}^{M}\left\|z_{n}^{s}\right\|_{L^{2}(\Omega)}=k \sum_{s=1}^{M}\left\|z_{n}^{s}\right\|_{L^{2}(\Omega)}
$$

where we used that $y_{n}^{0}=0$ as $B\left[u_{n}^{0}, v_{n}\right]=B\left[u(0), v_{n}\right]=B\left[w_{n}(0), v_{n}\right]$ for all $v_{n} \in S_{n}$. From (61) we then have

$$
\begin{gathered}
\max _{0 \leq j \leq M}\left\|y_{n}^{j}\right\|_{L^{2}(\Omega)} \leq C T h^{2}\left\|f_{t}\right\|_{L^{\infty}\left(0, T ; L^{2}(\Omega)\right)}+\left(C h^{2}+k\right) T\left\|u_{t t}\right\|_{L^{\infty}\left(0, T ; L^{2}(\Omega)\right)} \\
\leq C^{*}\left(h^{2}+k\right)\left(\left\|f^{\prime}(0)-P g\right\|_{\hat{\mathcal{K}}_{a+1}^{2}(\Omega)}+\left\|f_{t}\right\|_{L^{\infty}\left(0, T ; L^{2}(\Omega)\right)}\right. \\
\left.\quad+\left\|f_{t t}\right\|_{L^{2}\left(0, T ;\left(H_{\mathrm{D}}^{1}(\Omega)\right)^{\prime}\right)}+\|g\|_{\hat{\mathcal{K}}_{a+1}^{2}(\Omega)}\right)
\end{gathered}
$$

This bound implies

$$
\max _{0 \leq j \leq M}\left\|u_{n}^{j}-w_{n}(k j)\right\|_{L^{2}(\Omega)}=O\left(h^{2}+k\right)
$$

Integrating equation (56a) from 0 to $k j$ and combining it with the above bound, via the triangle inequality, then gives the desired $L^{2}$ estimate on the FEM approximation:

$$
\max _{0 \leq j \leq M}\left\|u_{n}^{j}-u(k j)\right\|_{L^{2}(\Omega)}=O\left(h^{2}+k\right) .
$$

Next we turn to the $H^{1}$ estimate. First, from (62) we have

$$
C k\left\|y_{n}^{j+1}\right\|_{H_{\mathrm{D}}^{1}(\Omega)}^{2} \leq k B\left[y_{n}^{j+1}, y_{n}^{j+1}\right]=\left(y_{n}^{j}+k z_{n}^{j+1}-y_{n}^{j+1}, y_{n}^{j+1}\right) \text {. }
$$

We estimate the right-hand side using (63), so that 


$$
\begin{array}{r}
\max _{0 \leq j \leq M}\left\|y_{n}^{j}\right\|_{H_{\mathrm{D}}^{1}(\Omega)} \leq \frac{C}{\sqrt{k}} \max _{0 \leq j \leq M}\left\|y_{n}^{j}\right\|_{L^{2}(\Omega)}+C \max _{0 \leq j \leq M}\left\|y_{n}^{j}\right\|_{L^{2}(\Omega)}^{1 / 2}\left\|z_{n}^{j}\right\|_{L^{2}(\Omega)}^{1 / 2} \\
=O\left(\frac{h^{2}+k}{\sqrt{k}}\right) .
\end{array}
$$

We integrate (56b) from 0 to $k j$ to obtain $\left\|u(j k)-w_{n}(j k)\right\|_{H_{\mathrm{D}}^{1}(\Omega)}=O(h)$. Finally, recall that $y_{n}^{j}=u_{n}^{j}-w_{n}(j k)$ and combine the two previous estimates using the triangle inequality again to conclude that

$$
\max _{0 \leq j \leq M}\left\|u_{n}^{j}-u(k j)\right\|_{H_{\mathrm{D}}^{1}(\Omega)}=O\left(h+\frac{h^{2}+k}{\sqrt{k}}\right) .
$$

Remark 6 If we we use quadratic elements, that is, we fix $m=2$, we obtain the improved rate:

$$
\begin{aligned}
& \max _{0 \leq j \leq M}\left\|u_{n}^{j}-u(k j)\right\|_{L^{2}(\Omega)}=O\left(h^{3}+k\right), \\
& \max _{0 \leq j \leq M}\left\|u_{n}^{j}-u(k j)\right\|_{H_{\mathrm{D}}^{1}(\Omega)}=O\left(h^{2}+\frac{h^{3}+k}{\sqrt{k}}\right) .
\end{aligned}
$$
(58).

The proof is similar to the case $m=1$, we only need to exploit higher regularity in

\section{Numerical Tests}

We implement the scheme presented in the Sect. 6 with three numerical tests. In these tests, we employ quadratic elements and use graded meshes, using the refinement strategy described in Sect. 6, taken from [32].

In the examples we present, the exact solution $u$ is unknown, so we compute the convergence rates empirically a posteriori by comparing the approximate solutions for two consecutive refinements in space and/or time. For each example below, we let the mesh size of our initial triangulation $\mathcal{T}_{0}$ be $h=2^{-2}$, so $\mathcal{T}_{n}$ will have maximal mesh size $h=2^{-n-2}$. In addition, for each triangulation, we let the time step size $k=4 h^{3}$, so that we can expect an optimal error of order $O\left(h^{\frac{3}{2}}\right)$ in $L^{\infty} H^{1}$ from the second equation in (64). Since the time and mesh sizes are related, we denote the approximate solution simply by $u_{n}^{h}$. Then theoretically

$$
\lim _{n \rightarrow \infty} \log _{2} e_{n}=\lim _{n \rightarrow \infty} \frac{\left\|u_{n-1}^{h}-u_{n}^{h}\right\|_{H_{\mathrm{D}}^{1}(\Omega)}}{\left\|u_{n}^{h}-u_{n+1}^{h}\right\|_{H_{\mathrm{D}}^{1}(\Omega)}} \geq 2 \sqrt{2} \approx 2.828,
$$

where the lower bound may not be optimal. This choice of time stepping is convenient for computing the convergence rate, however it is computationally intensive and we 
Fig. 5 Example: non-smooth interface

Fig. 6 Initial triangulation $\mathcal{T}_{0}$

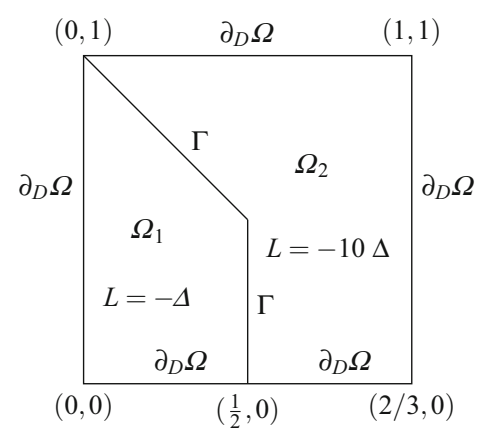

$(0,1)$

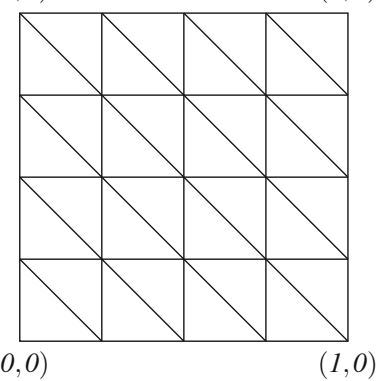

are able to refine the mesh only a few times (all calculations were done on a laptop computer). The numerical implementation is not the main focus of our work.

\subsection{Domain with Non-smooth Interface}

In the first test, we let the domain be a square that is divided into two polygons by the interface. Specifically, let $\Omega=(0,1) \times(0,1), T=[0,1], \partial \Omega=\partial_{\mathrm{D}} \Omega$, with the interface $\Gamma=\left\{(1-y, y) \mid y \in\left(\frac{1}{2}, 1\right)\right\} \cup\left\{\left(\frac{1}{2}, y\right) \mid y \in\left(0, \frac{1}{2}\right]\right\}$. We remark that $\Gamma$ has an interior vertex at $\left(\frac{1}{2}, \frac{1}{2}\right)$. Then the two subdomains are given by

$$
\Omega_{1}=\left\{(x, y) \mid x \in\left(0, \frac{1}{2}\right), y \in(0,1-x)\right\}, \quad \Omega_{2}=\Omega \backslash \Omega_{1},
$$

while we choose the operator

$$
L= \begin{cases}-\Delta & \text { on } \Omega_{1} \\ -10 \Delta & \text { on } \Omega_{2}\end{cases}
$$

with data $g(x, y) \equiv 0$ and $f(x, y, t)=\sin (\pi x) \sin (\pi y) t$ for all $(x, y, t) \in$ $(0,1) \times(0,1) \times[0,1]$, so we solve for times $0 \leq t \leq T=1$ (Figs. 5, 6).

The initial mesh $\mathcal{T}_{0}$ is uniform with mesh size $h=0.25$. We note that there are 6 vertices on the boundary of $\Omega$ and the interface $\Gamma$, where the solution $u$ is singular. By 

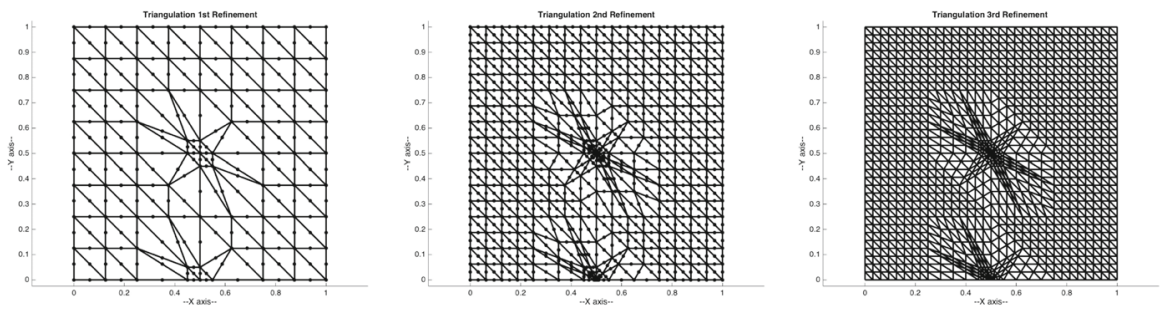

Fig. 7 First 3 mesh refinements $\mathcal{T}_{1}, \mathcal{T}_{2}, \mathcal{T}_{3}$ after the initial uniform mesh

Table 1 Errors: domain with non-smooth interface

\begin{tabular}{lllll}
\hline & $\left\|u_{0}^{h}-u_{1}^{h}\right\|_{H_{\mathrm{D}}^{1}(\Omega)}$ & $\left\|u_{1}^{h}-u_{2}^{h}\right\|_{H_{\mathrm{D}}^{1}(\Omega)}$ & $\left\|u_{2}^{h}-u_{3}^{h}\right\|_{H_{\mathrm{D}}^{1}(\Omega)}$ & $\left\|u_{3}^{h}\right\|_{H_{\mathrm{D}}^{1}(\Omega)}$ \\
\hline$t=0.0$ & 0 & 0 & 0 & 0 \\
$t=0.25$ & $1.202 e^{-5}$ & $7.166 e^{-7}$ & $1.189 e^{-7}$ & $7.261 e^{-5}$ \\
$t=0.5$ & $5.248 e^{-5}$ & $3.113 e^{-6}$ & $5.076 e^{-7}$ & $3.137 e^{-4}$ \\
$t=1.0$ & $2.191 e^{-4}$ & $1.295 e^{-5}$ & $2.037 e^{-6}$ & $1.305 e^{-3}$ \\
\hline
\end{tabular}
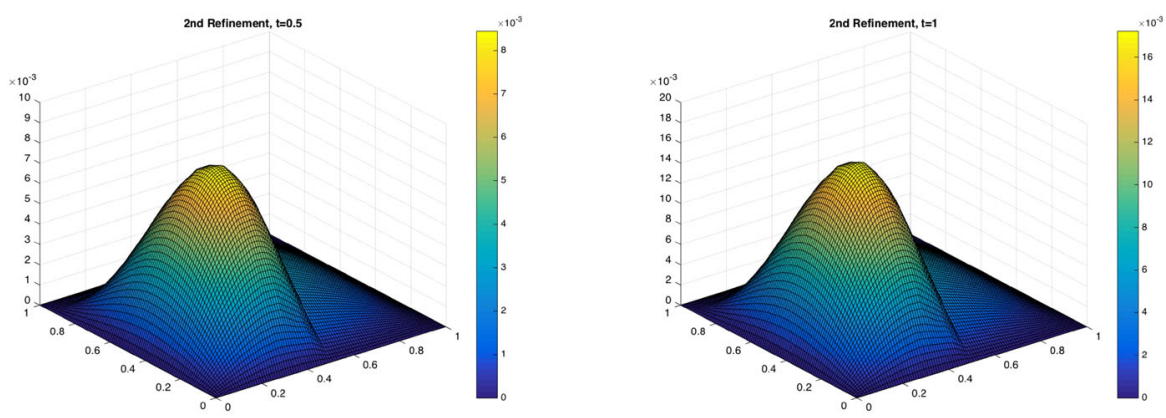

Fig. 8 Numerical solution $u_{2}^{h} \in S_{2}: S\left(\mathcal{T}_{2}, 2\right)$ at time $t=0.5$ and $t=1$

Theorem 5, at each vertex of the unit square, the singularity constant $\eta_{Q}=2$, while at the vertices $\left(\frac{1}{2}, 0\right)$ and $\left(\frac{1}{2}, \frac{1}{2}\right), \eta_{Q}$ equals 1 and $0.839<1$, respectively. Consequently at each corner of the unit square, the refinement ratio $\kappa_{Q}=2^{-m} \eta_{Q}=1 / 2$, and no grading is needed as expected. At the vertices $\left(\frac{1}{2}, 0\right)$ and $\left(\frac{1}{2}, \frac{1}{2}\right), \kappa_{Q}=1 / 4=0.25$ and $\kappa_{Q}=0.839 / 4=0.210$, respectively, which gives the graded mesh in Fig. 7 .

We tabulate the errors for the first three refinements (again we take the mesh size $h=$ $2^{-n-2}$ and time step size $k=4 h^{3}=2^{-3 n-4}$ for $\left.n=1,2,3\right)$ in Table 1. From Table 1 , we check that $e_{1}=2.191 e^{-4} / 1.295 e^{-5} \approx 17$, while $e_{2}=1.295 e^{-5} / 2.037 e^{-6} \approx 6$ at $t=1$. The computational costs increase significantly for $n>3$ (Fig. 8). 
Fig. 9 Example:

Neumann-Neumann vertices

Fig. 10 Initial triangulation $\mathcal{T}_{0}$

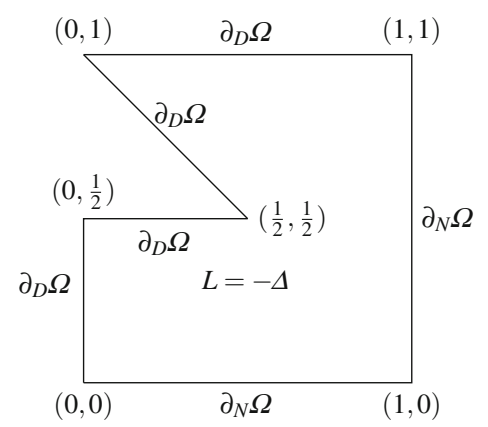

$(0,1)$

$(1,1)$

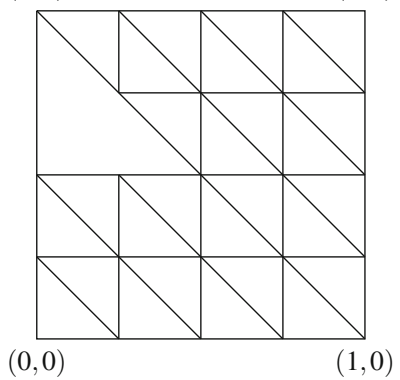

\subsection{Domain with Neumann-Neumann Vertices}

We turn to our second example, in which the domain is non-convex and contains a Neumann-Neumann vertex. We let the domain $\Omega$ be the unit square $(0,1) \times(0,1)$ minus the triangle with vertices $(0,1),\left(0, \frac{1}{2}\right)$ and $\left(\frac{1}{2}, \frac{1}{2}\right)$. Moreover, we choose $L=$ $-\Delta$ on $\Omega$ so that there is no interface. On the boundary, we impose Neumann condition on the sides from $(0,0)$ to $(1,0)$ and from $(1,0)$ to $(1,1)$, and impose Dirichlet condition on the other sides. As in the previous example, we take $T=1$ and the data $g(x, y) \equiv 0$ and $f(x, y, t)=\sin (\pi x) \sin (\pi y) t$ for all $(x, y, t) \in(0,1) \times(0,1) \times$ $[0,1]$ (Figs. 9, 10).

As in the previous case, we calculate $\eta_{Q}$ using Theorem 5. As a result, we can refine uniformly at the vertices $(0,1),(1,0)$ and $\left(0, \frac{1}{2}\right)$. At the vertices $(0,0)$ and $(1,1)$ where the boundary condition changes from Dirichlet to Neumann, the refinement ratio is $\kappa_{Q}=2^{-2} \eta=0.25$, while at the vertex $\left(\frac{1}{2}, \frac{1}{2}\right), \kappa_{Q}=2^{-2} \times \frac{7}{4}=0.143$ (see Fig. 11).

We tabulate the error again for the first three refinements in Table 2, and compute $e_{1}=3.100 e^{-3} / 7.263 e^{-4} \approx 4.27$, and $e_{2}=7.263 e^{-4} / 1.883 e^{-4} \approx 3.86$ at time $t=$ 1. The error is stabilizing at the theoretical rate faster, as no interface is present (Fig. 12).

\subsection{Problem with High Contrast Across Interface}

In the last test, we let the domain $\Omega$ be the unit square partitioned equally into two sub-rectangles by an interface. We impose Dirichlet conditions on the entire boundary of $\Omega$ and solve again for $0 \leq t \leq T=1$. The two subdomains are given by: 

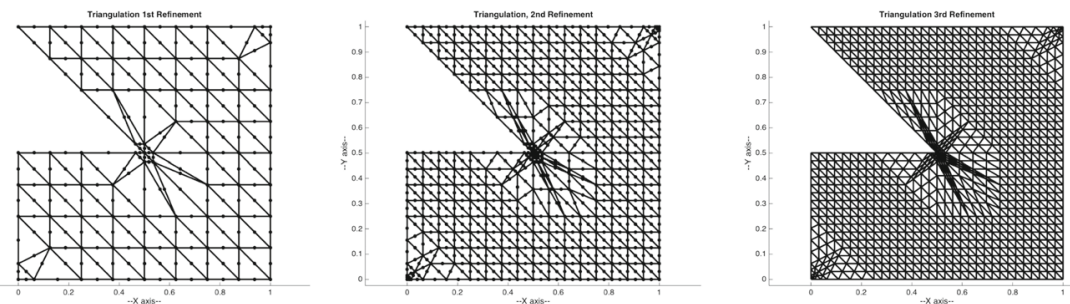

Fig. 11 First 3 mesh refinements $\mathcal{T}_{1}, \mathcal{T}_{2}, \mathcal{T}_{3}$ after the initial uniform mesh

Table 2 Errors: domain with Neumann-Neumann vertices

\begin{tabular}{lllll}
\hline & $\left\|u_{0}^{h}-u_{1}^{h}\right\|_{H_{\mathrm{D}}^{1}(\Omega)}$ & $\left\|u_{1}^{h}-u_{2}^{h}\right\|_{H_{\mathrm{D}}^{1}(\Omega)}$ & $\left\|u_{2}^{h}-u_{3}^{h}\right\|_{H_{\mathrm{D}}^{1}(\Omega)}$ & $\left\|u_{3}^{h}\right\|_{H_{\mathrm{D}}^{1}(\Omega)}$ \\
\hline$t=0.0$ & 0 & 0 & 0 & 0 \\
$t=0.25$ & $9.012 e^{-5}$ & $2.447 e^{-5}$ & $7.520 e^{-6}$ & $3.028 e^{-4}$ \\
$t=0.5$ & $5.834 e^{-4}$ & $1.448 e^{-4}$ & $4.011 e^{-5}$ & $1.878 e^{-3}$ \\
$t=1.0$ & $3.100 e^{-3}$ & $7.263 e^{-4}$ & $1.883 e^{-4}$ & $9.737 e^{-3}$ \\
\hline
\end{tabular}
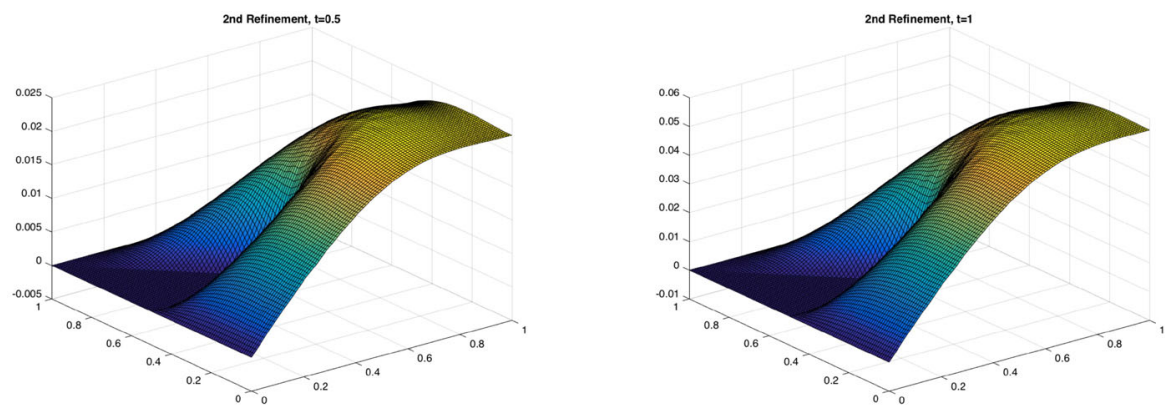

Fig. 12 Numerical solution $u_{2}^{h} \in S_{2}: S\left(\mathcal{T}_{2}, 2\right)$ at time $t=0.5$ and $t=1$

$$
\Omega_{1}=\left(0, \frac{1}{2}\right) \times(0,1), \quad \Omega_{2}=\left(\frac{1}{2}, 1\right) \times(0,1)
$$

The operator $L$ is chosen to be

$$
L= \begin{cases}-\Delta & \text { on } \Omega_{1} \\ -100 \Delta & \text { on } \Omega_{2}\end{cases}
$$

Hence a high contrast across the interface $\Gamma$ (Figs. 13, 14). To meet the compatibility conditions across the interface, we let the data $f \equiv 0$, and

$$
g(x, y)= \begin{cases}y(1-y)\left(\frac{31300}{808} x-200 x^{3}+100 x^{4}\right) & \text { on } \Omega_{1} \\ y(1-y)\left(\frac{1303}{808}(1-x)-2(1-x)^{3}+(1-x)^{4}\right) & \text { on } \Omega_{2}\end{cases}
$$


Fig. 13 Example: high contrast operator

Fig. 14 Initial triangulation $\mathcal{T}_{0}$

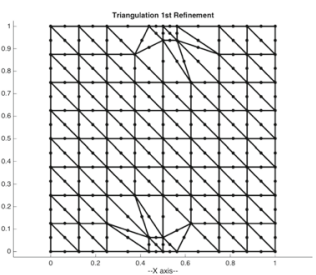

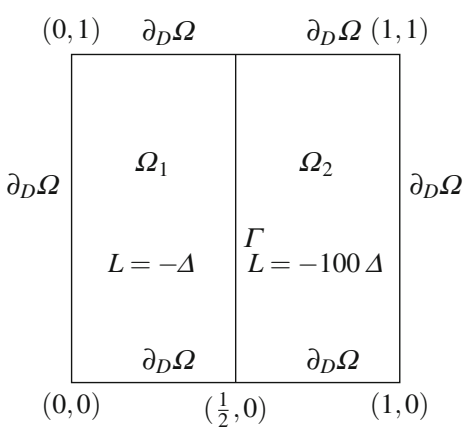

$(0,1)$

$(1,1)$
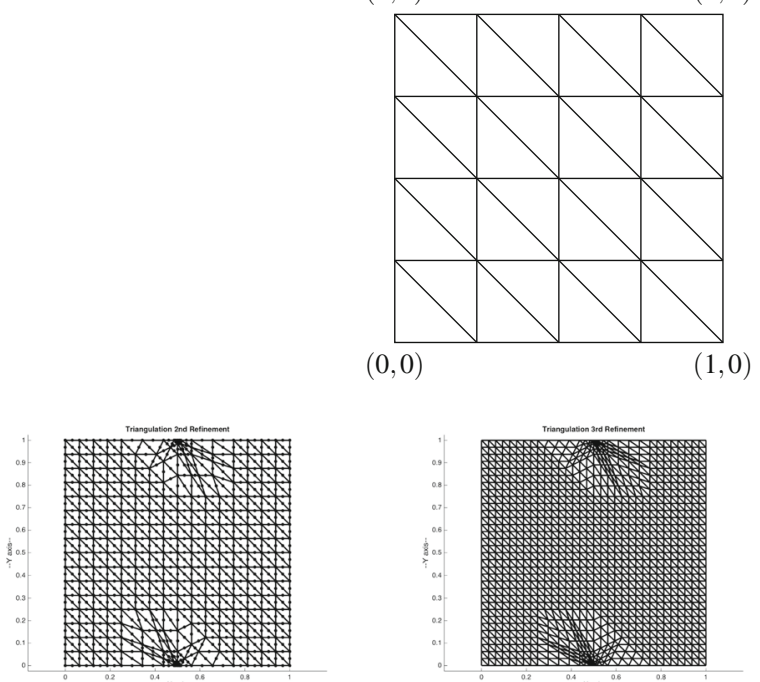

Fig. 15 First 3 mesh refinements $\mathcal{T}_{1}, \mathcal{T}_{2}, \mathcal{T}_{3}$ after the initial uniform mesh

Table 3 Table: problem with high contrast operator

\begin{tabular}{lllll}
\hline & $\left\|u_{0}^{h}-u_{1}^{h}\right\|_{H_{\mathrm{D}}^{1}(\Omega)}$ & $\left\|u_{1}^{h}-u_{2}^{h}\right\|_{H_{\mathrm{D}}^{1}(\Omega)}$ & $\left\|u_{2}^{h}-u_{3}^{h}\right\|_{H_{\mathrm{D}}^{1}(\Omega)}$ & $\left\|u_{3}^{h}\right\|_{H_{\mathrm{D}}^{1}(\Omega)}$ \\
\hline$t=0.0$ & $9.401 e^{-1}$ & $1.250 e^{-2}$ & $2.381 e^{-03}$ & 20.31 \\
$t=0.25$ & $3.060 e^{-4}$ & $1.491 e^{-8}$ & $9.717 e^{-10}$ & $1.736 e^{-10}$ \\
$t=0.5$ & $4.588 e^{-9}$ & $2.410 e^{-17}$ & $4.505 e^{-20}$ & $1.425 e^{-22}$ \\
$t=1.0$ & $9.972 e^{-19}$ & $3.268 e^{-35}$ & $9.681 e^{-41}$ & $8.524 e^{-43}$ \\
\hline
\end{tabular}

We check again using Theorem 5 that at the four vertices of the unit square, $\kappa_{Q}=0.5$ so no special grading is needed, while where the interface meets the boundary, the points $\left(\frac{1}{2}, 0\right)$ and $\left(\frac{1}{2}, 1\right), \eta_{Q}=1$, so the refinement ratio is $\kappa_{Q}=2^{-2}=0.25$. The first three mesh refinements are shown in Fig. 15.

The corresponding error in the numerical solution is given in Table 3. 

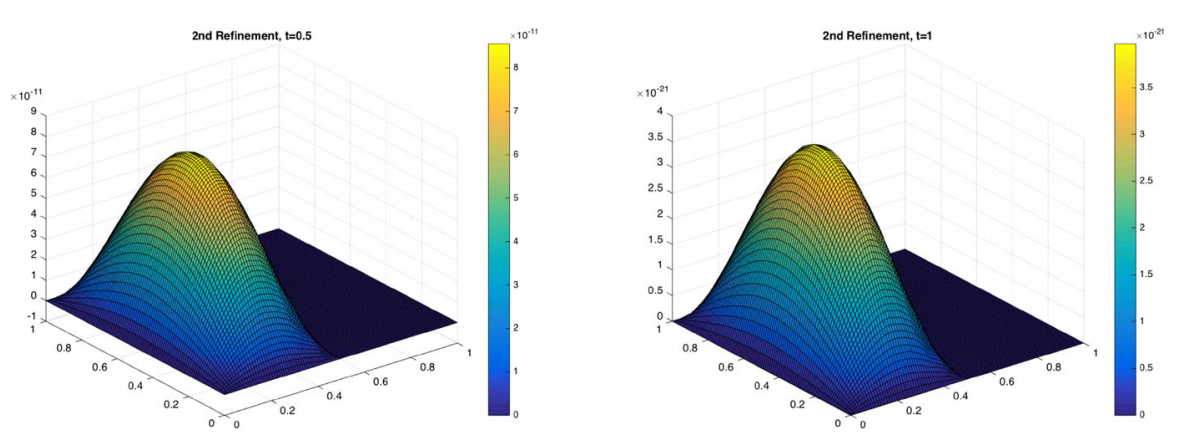

Fig. 16 Numerical solution $u_{2}^{h} \in S_{2}: S\left(\mathcal{T}_{2}, 2\right)$ at time $t=0.5$ and $t=1$

From this table, we compute $e_{1}=9.972 e^{-19} / 3.268 e^{-35} \approx 3.05 e^{16}$ and $e_{2}=$ $=3.268 e^{-35} / 9.681 e^{-41} \approx 3.38 e^{5}$ at $t=1$. So, the relative error is large. Significantly more refinements are needed to achieve the theoretical rate. The large numerical error stems from the high contrast across the interface $\Gamma$, which has an effect on the time derivative as well. Further numerical tests have shown that a mesh size $h<\frac{1}{200}$ is needed to have an error of $<10 \%$ (Fig. 16).

Acknowledgements The authors thank Serge Nicaise and Victor Nistor for useful discussions.

Funding Yajie Zhang was partially supported by the US National Science Foundation grant DMS1312727.Anna L. Mazzucato was partially suppported the US National Science Foundation grants DMS-1312727, DMS-1615457, and DMS-1909103.

\section{References}

1. Abels, H., Wilke, M.: Well-posedness and qualitative behaviour of solutions for a two-phase NavierStokes-Mullins-Sekerka system. Interfaces Free Bound. 15(1), 39-75 (2013). https://doi.org/10.4171/ $\mathrm{IFB} / 294$

2. Adler, J.H., Nistor, V.: Graded mesh approximation in weighted Sobolev spaces and elliptic equations in 2D. Math. Comput. 84(295), 2191-2220 (2015). https://doi.org/10.1090/S0025-5718-2015-029342

3. Agranovich, M.S.: Sobolev spaces, their generalizations and elliptic problems in smooth and Lipschitz domains. In: Springer Monographs in Mathematics. Springer, Cham (2015)

4. Alberty, J., Carstensen, C., Funken, S.A.: Remarks around 50 lines of Matlab: short finite element implementation. Numer. Algorithms 20(2-3), 117-137 (1999). https://doi.org/10.1023/A:1019155918070

5. Amann, H.: Maximal regularity of parabolic transmission problems. J. Evol. Equ. (2020). https://doi. org/10.1007/s00028-020-00612-y

6. Ammann, B., Nistor, V.: Weighted sobolev spaces and regularity for polyhedral domains. Comput. Methods Appl. Mech. Eng. 196, 37-40 (2007)

7. Arnold, D.N.: Lecture Notes on Numerical Analysis of Partial Differential Equation. Springer, Berlin (2011)

8. Bacuta, C., Mazzucato, A.L., Nistor, V., Zikatanov, L.: Interface and mixed boundary value problems on n-dimensional polyhedral domains. Doc. Math. 15, 687-745 (2010)

9. Bacuta, C., Nistor, V., Zikatanov, L.: Anisotropic regularity and optimal rates of convergence for the finite element method in three dimensional polyhedral domains. Advances in Mathematics (2013)

10. Bacuta, C., Nistor, V., Zikatanov, L.T.: Improving the rate of convergence of high-order finite elements on polyhedra. II. Mesh refinements and interpolation. Numer. Funct. Anal. Optim. 28(7-8), 775-824 (2007). https://doi.org/10.1080/01630560701493263 
11. Bourlard, M., Dauge, M., Lubuma, M.S., Nicaise, S.: Coefficients of the singularities for elliptic boundary value problems on domains with conical points. III. Finite element methods on polygonal domains. SIAM J. Numer. Anal. 29(1), 136-155 (1992)

12. Băcuţă, C., Nistor, V., Zikatanov, L.T.: Improving the rate of convergence of 'high order finite elements' on polygons and domains with cusps. Numer. Math. 100(2), 165-184 (2005). https://doi.org/10.1007/ s00211-005-0588-3

13. Caffarelli, L.A., Peral, I.: On $W^{1, p}$ estimates for elliptic equations in divergence form. Commun. Pure Appl. Math. 51(1), 1-21 (1998)

14. Chipot, M., Kinderlehrer, D., Vergara-Caffarelli, G.: Smoothness of linear laminates. Arch. Ratl. Mech. Anal. 96(1), 81-96 (1986). https://doi.org/10.1007/BF00251414

15. Costabel, M., Dauge, M.: General edge asymptotics of solutions of second-order elliptic boundary value problems. I, II. Proc. R. Soc. Edinb. Sect. A 123(1), 109-155 (1993)

16. Costabel, M., Dauge, M.: Crack singularities for general elliptic systems. Math. Nachr. 235, 29-49 (2002)

17. Costabel, M., Dauge, M., Nicaise, S.: Singularities of maxwell interface problems. Math. Model. Numer. Anal. 33(3), 627-649 (1999)

18. Costabel, M., Dauge, M., Nicaise, S.: Analytic regularity for linear elliptic systems in polygons and polyhedra. Math. Models Methods Appl. Sci. 22(8), 1250015 (2012)

19. Dauge, M.: Elliptic Boundary Value Problems on Corner Domains. Smoothness and Asymptotics of Solutions, vol. 123. Springer-Verlag, Berlin (1988)

20. Disser, K., ter Elst, A.F.M., Rehberg, J.: Hölder estimates for parabolic operators on domains with rough boundary. Ann. Sc. Norm. Super. Pisa Cl. Sci. (5) 17(1), 65-79 (2017)

21. Druet, P.E.: Global lipschitz continuity for elliptic transmission problems with a boundary intersecting interface. Math. Bohem. 138(2), 185-224 (2013)

22. Druet, P.E.: Regularity of second derivatives in elliptic transmission problems near an interior regular multiple line of contact. Math. Methods Appl. Sci. 41(16), 6457-6479 (2018). https://doi.org/10.1002/ mma.5170

23. Elschner, J., Rehberg, J., Schmidt, G.: Optimal regularity for elliptic transmission problems including $C^{1}$ interfaces. Interfaces Free Bound. 9(2), 233-252 (2007). https://doi.org/10.4171/IFB/163

24. Escauriaza, L., Fabes, E.B., Verchota, G.: On a regularity theorem for weak solutions to transmission problems with internal Lipschitz boundaries. Proc. Am. Math. Soc. 115(4), 1069-1076 (1992). https:// doi.org/10.2307/2159357

25. Escauriaza, L., Mitrea, M.: Transmission problems and spectral theory for singular integral operators on Lipschitz domains. J. Funct. Anal. 216(1), 141-171 (2004). https://doi.org/10.1016/j.jfa.2003.12. 005

26. Escauriaza, L., Seo, J.K.: Regularity properties of solutions to transmission problems. Trans. Am. Math. Soc. 338(1), 405-430 (1993). https://doi.org/10.2307/2154462

27. Evans, L.C.: Partial differential equations. In: Graduate Studies in Mathematics, vol. 19, second edn. American Mathematical Society, Providence, RI (2010). https://doi.org/10.1090/gsm/019

28. Haller-Dintelmann, R., Rehberg, J.: Maximal parabolic regularity for divergence operators including mixed boundary conditions. J. Differ. Equ. 247(5), 1354-1396 (2009). https://doi.org/10.1016/j.jde. 2009.06.001

29. Kohr, M., Pintea, C., Wendland, W.L.: Dirichlet-transmission problems for general Brinkman operators on Lipschitz and $C^{1}$ domains in Riemannian manifolds. Discrete Contin. Dyn. Syst. Ser. B 15(4), 9991018 (2011). https://doi.org/10.3934/dcdsb.2011.15.999

30. Kondratev, V.A.: Boundary value problems for elliptic equations in domains with conical or angular points, vol. 16, second edn. Tr. Mosk. Mat. Obs (1967)

31. Ladyzenskaja, O.A., Rivkind, V.J., Uralceva, N.N.: Solvability of diffraction problems in the classical sense. Trudy Mat. Inst. Steklov. 92, 116-146 (1966)

32. Li, H., Mazzucato, A., Nistor, V.: Analysis of the finite element method for transmission/mixed boundary value problems on general polygonal domains. Electron. Trans. Numer. Anal. 37, 41-69 (2010)

33. Li, H., Nistor, V.: Graded meshes on domains of polygonal structures. Recent Adv. Sci. Comput. Appl. 586, 239 (2013)

34. Li, H., Nistor, V., Qiao, Y.: Uniform shift estimates for transmission problems and optimal rates of convergence for the parametric finite element method. In: Numerical Analysis and Its Applications (2013) 
35. Li, Y., Nirenberg, L.: Estimates for elliptic systems from composite material. pp. 892-925 (2003). https://doi.org/10.1002/cpa.10079. (Dedicated to the memory of Jürgen K. Moser)

36. Lions, J., Magenes, E.: Non-homogeneous Boundary Value Problems and Applications, vol. 1. Springer-Verlag, New York (1972)

37. Mazya, V., Kozlov, V., Rossmann, J.: Elliptic Boundary Value Problems in Domains with Point Singularities, vol. 52. Mathematical Surveys and Monographs, Providence, RI (1997)

38. Mazya, V.G., Plamenevskii, B.A.: The first boundary value problem for classical equations of mathematical physics in domains with piecewise smooth boundaries. II. Z. Anal. Anwendungen 2(6), 335-359 (1983)

39. Mazzucato, A., Nistor, V.: Well-posedness and regularity for the elasticity equation with mixed boundary conditions on polyhedral domains and domains with cracks. Arch. Ration. Mech. Anal. 195(1), 25-73 (2010)

40. Mercier, D., Nicaise, S.: Regularity results of stokes/lamé interface problems. Math. Nachr. 285(2-3), 332-348 (2012)

41. Milovanović Jeknić, Z.: Parabolic-hyperbolic transmission problem in disjoint domains. Filomat 32(20), 6911-6920 (2018). https://doi.org/10.2298/fil1820911m

42. Mitrea, I., Ott, K.: Counterexamples to the well-posedness of $L^{p}$ transmission boundary value problems for the Laplacian. Proc. Am. Math. Soc. 135(7), 2037-2043 (2007). https://doi.org/10.1090/S00029939-07-08686-8

43. Mullins, W.W., Sekerka, R.F.: Morphological stability of a particle growing by diffusion or heat flow. In: Fundamental Contributions to the Continuum Theory of Evolving Phase Interfaces in Solids, pp. 75-81. Springer, Berlin (1999)

44. Nicaise, S.: Interface problems and coefficients of the singularities. Bull. Soc. Math. Belg. Sér. 41(1) (1989)

45. Nicaise, S.: Interface problems and coefficients of the singularities. Commun. Partial Differ. Equ.15(10) (1990)

46. Nicaise, S., Sandig, A.M.: General interface problems. i, ii. Math. Methods Appl. Sci. 17 (1994)

47. Pazy, A.: Semigroups of linear operators and applications to partial differential equations. In: Applied Mathematical Sciences, vol. 44, second edn (1983)

48. Prüss, J., Simonett, G., Wilke, M.: The Rayleigh-Taylor instability for the Verigin problem with and without phase transition. NoDEA Nonlinear Differ. Equ. Appl. 26(3), 35 (2019). https://doi.org/10. 1007/s00030-019-0564-8

49. Rauchecker, M.: Strong solutions to the Stefan problem with Gibbs-Thomson correction and boundary contact (2020) arXiv:2001.06438

50. Roŭtberg, B.Y.: Transmission problems in domains with nonsmooth boundaries. Dopov. Nats. Akad. Nauk Ukraïni 3, 15-20 (1996)

51. Rol̆tberg, J.A., Seftel, Z.G.: On equations of elliptic type with discontinuous coefficients. Dokl. Akad. Nauk SSSR 146, 1275-1278 (1962)

52. Xiong, J., Bao, J.: Sharp regularity for elliptic systems associated with transmission problems. Potential Anal. 39(2), 169-194 (2013)

53. Zhang, Y.: Transmission problems for parabolic equations and applications to the finite element method. Ph.D. thesis, Penn State University, University Park, PA (2017). https://etda.libraries.psu.edu/catalog/ 14527 yxz 170

Publisher's Note Springer Nature remains neutral with regard to jurisdictional claims in published maps and institutional affiliations. 